\title{
An Adaptive Transmission Scheme for Cognitive Decode-and-Forward Relaying Networks: Half Duplex, Full Duplex, or No Cooperation
}

\author{
Edgar Eduardo Benítez Olivo, Member, IEEE, Diana Pamela Moya Osorio, Member, IEEE, \\ Hirley Alves, Member, IEEE, José Cândido Silveira Santos Filho, Member, IEEE, \\ and Matti Latva-aho, Member, IEEE
}

\begin{abstract}
We propose an adaptive transmission scheme for cognitive decode-and-forward relaying networks, whereby, before each communication process, one out of three transmission modes is dynamically selected in order to maximize the instantaneous capacity of the system, namely, half-duplex (HD) relaying, full-duplex (FD) relaying, or direct transmission with no cooperation. The following key issues, relevant to underlay spectrum sharing and cooperative relaying, are considered: 1) the overall transmit power at the secondary network is constrained by both the maximum tolerable interference at the primary receiver and the maximum transmit power available at the secondary nodes; 2) under FD operation, the secondary relay is subject to residual self-interference, which is modeled as a fading channel; and 3) the signals coming from the secondary source and relay are handled at the secondary destination via maximal-ratio combining, in the HD relaying mode, and via a joint-decoding technique, in the FD relaying mode. We derive an exact analytical expression for the outage probability of the proposed scheme. Then, an approximate closed-form expression is proposed, and a corresponding asymptotic expression is derived. Monte Carlo simulations are run to validate the accuracy of the presented mathematical analysis and to showcase the tightness of the proposed approximation.
\end{abstract}

Index Terms-Cognitive radio, cooperative diversity, decode-and-forward, full-duplex relaying, half-duplex relaying, outage probability, self-interference, underlay spectrum sharing.

Manuscript received November 14, 2015; revised February 26, 2016; accepted April 19, 2016. Date of publication May 3, 2016; date of current version August 10, 2016. This work was supported in part by the Brazilian Ministry of Education Coordenação de Aperfeiçoamento de Pessoal de Nível Superior, in part by the Finnish Centre for International Mobility, and in part by the Tekes and Academy of Finland. The associate editor coordinating the review of this paper and approving it for publication was A. Wyglinski.

E. E. Benítez Olivo is with the School of Telecommunications Engineering, São Paulo State University, São João da Boa Vista 13876-750, Brazil, and also with the Department of Communications, School of Electrical and Computer Engineering, University of Campinas, Campinas 13083-852, Brazil (e-mail: edgar.olivo@sjbv.unesp.br).

D. P. Moya Osorio is with the Department of Electrical Engineering, Federal University of São Carlos, São Carlos 13565-905, Brazil (e-mail: dianamoya@ufscar.br).

H. Alves and M. Latva-aho are with the Centre for Wireless Communications, University of Oulu, Oulu 90014, Finland (e-mail: halves@ee.oulu.fi; matla@ee.oulu.fi).

J. C. S. Santos Filho is with the Department of Communications, School of Electrical and Computer Engineering, University of Campinas, Campinas 13083-852, Brazil (e-mail: candido@decom.fee.unicamp.br).

Digital Object Identifier 10.1109/TWC.2016.2562109

\section{INTRODUCTION}

$\mathbf{T}$ HE concept of spectrum sharing in cognitive radio networks has been envisioned as one of the key technologies to cope with the problems of spectrum shortage and underutilization [1], [2], by enabling secondary (unlicensed/cognitive) users to opportunistically access, under controlled or no interference, the frequency bands allocated to primary (licensed) users, without compromising the target quality of service of the licensed network [3]-[6]. Depending on the spectrum-access strategy, the operation of a cognitive radio network can be classified as underlay, overlay, and interweave [7]. Herein, we focus on an underlay spectrum-sharing network, in which the secondary users (SUs) are allowed to concurrently access the licensed band, provided that the resulting interference on the primary users (PUs) remains below a given tolerated level, termed as interference temperature [4]. To do so, SUs have to adaptively adjust their transmit power according to the channel state information (CSI) of the interference links from the secondary to the primary network, so as to meet the interference power constraint at PU receivers. As a consequence, the performance of an underlay cognitive radio network is subordinated to the channel statistics of the interference links from SUs to PUs, thereby jeopardizing the communication reliability and cell coverage of the secondary network. To tackle this concern, cooperative relaying ${ }^{1}$ has been introduced in cognitive radio networks as a promising approach to boost the performance of secondary transmissions, without increasing the transmit power, while meeting the interference power constraint at PUs.

According to the relay operation, the relaying techniques can be classified in two basic modes, namely, half-duplex (HD) relaying and full-duplex (FD) relaying. In the HD relaying mode, the relay receives and forwards signals in orthogonal channels (e.g., orthogonal time slots), thus consuming twice the channel resources required by direct transmission (DT) and, consequently, incurring a loss of spectral efficiency [9].

\footnotetext{
${ }^{1}$ The basic idea of cooperative relaying is to leverage the antennas available at neighboring nodes (a.k.a. relays) in the network, with the aim of emulating a distributed antenna array, and thus creating a new form of spatial diversity, referred to as cooperative diversity [8]-[10]. This way, even single-antenna nodes are able to achieve the same benefits offered by multiple-input multipleoutput (MIMO) systems, namely, spatial degree of freedom, increased spectral efficiency, and diversity [11].
} 
On the other hand, the FD relaying mode enables the relay to receive and forward signals concurrently on the same frequency band, thereby retrieving the spectral-efficiency loss inherent to HD relaying. However, the spectral-efficiency improvement of FD relaying comes at the expense of selfinterference at the relay, which is induced by the signal leakage from the relay output to its input [14], [21], thus worsening the system performance. In this respect, there have been substantial efforts in the design and implementation of different selfinterference mitigation techniques, by considering SISO and MIMO relay setups. These include the following: (i) passive suppression schemes, whereby the self-interference signal is suppressed in the propagation domain before being processed by the receiver circuitry, such as natural isolation, orthogonal polarization, antenna selection, beam selection, and null-space projection [12]-[16]; and (ii) active (digital/analog) cancellation schemes, whereby the self-interference signal is mitigated by subtracting a processed replica of the transmitted signal from the received signal [17]-[20]. However, because of practical implementation limitations and non-idealities, these techniques cannot guarantee a perfect self-interference mitigation. Then, FD relays may still suffer from a remaining level of selfinterference-referred to as residual self-interference (RSI) which cannot be regarded simply as Gaussian noise [18]. Indeed, the RSI is usually modeled as a fading channel and, as such, it can significantly impact the performance of the system, rendering its diversity order nil [21], [22].

Clearly, this poses a tradeoff between the spectral-efficiency loss and the self-interference problem inherent to the HD and FD relaying modes, respectively. In order to find a balance, some relaying schemes that adaptively operate in HD or FD mode have been proposed in the context of conventional relaying networks without spectrum sharing [21], [22]. For example, in [21], a hybrid FD/HD relaying scheme was introduced to maximize the instantaneous and average spectral efficiencies. In that work, the switching boundaries between the FD and HD relaying modes were provided, by considering both amplify-and-forward (AF) and decode-and-forward (DF) relaying protocols. Such a hybrid FD/HD relaying scheme is shown to achieve a significant performance improvement with respect to using either the HD or FD relaying mode alone. It was also shown that the FD relaying mode outperforms the HD relaying mode, as long as the RSI can be mitigated below the noise level. In turn, in [22], an optimal relay selection strategy, based on the hybrid FD/HD relaying policy in [21], was investigated in terms of outage probability. This hybrid relay-selection scheme is shown to be superior to the HD-based relay selection schemes, as well as to overcome the zero diversity-order problem of FD-based schemes.

In the context of cognitive relaying networks (CRNs) with underlay spectrum sharing, several scenarios have been investigated to show the advantages and limitations of cooperative relaying techniques, as well as the impact of PUs on the performance of the secondary network, by considering either HD or FD relaying. Certainly, most of the works in the related literature have been devoted to the performance analysis of HD-based CRNs, with the outage probability as one of its foremost performance metrics [25]-[33]. Those studies address a wide range of sophisticated scenarios in addition to the classical single-antenna triple-node cognitive relaying network setup [25], [26], which also leverage the benefits of other diversity techniques, including relay selection [27], [28], multiuser (destination) diversity [30], [31], multiantenna techniques (e.g., transmit beamforming/antenna selection and maximal-ratio/selection combining) [32], [33], among others. On the other hand, very few works have focused on FD-based CRNs [34]-[36]. Among them, in [34], the outage performance of a FD-DF relaying system under spectrum-sharing constraints has been investigated. Therein, an equal power allocation (EPA) scheme between the secondary source and relay was considered, which is limited by the interference power constraint at the PU. In addition, two main sources of interference at the secondary network were considered: the RSI at the secondary relay, which is inherent to the FD operation mode, and the interference from the secondary source at the secondary destination through the direct-link transmission, which is concurrent with the relaying transmission. In contrast to the scheme in [34], but similarly to the schemes in [23] and [24], proposed in the context of conventional relaying networks without spectrum sharing, in [35], the direct-link transmission is considered to convey useful information. Thus, an additional diversity path is obtained and, consequently, the outage performance is improved w.r.t. that attained by its counterpart in [34]. Hereafter, we refer to such a coding scheme-one that enables the secondary destination to jointly decode the signals coming from the secondary source and FD relay-as joint decoding (JD). ${ }^{2}$ On the other hand, a major practical concern with the schemes in [34] and [35] is that the maximum transmit power available at the secondary nodes is considered to be unbounded, as it only depends on the ratio between the interference power constraint and the channel state from the secondary node to the primary receiver. In [36], the outage probability for three relay selection policies based on FD-AF relaying was analyzed. Therein, the effects of the interference power constraint at the primary network and the maximum transmit power constraint at the secondary nodes, as well as the RSI, were considered. However, in that study, the impact of the direct-link transmission was disregarded.

Differently from all prior works concerning underlay CRNs, in which the secondary network is limited to operate in either HD or FD relaying mode, and in order to overcome the limitations inherent to each relaying mode, herein we propose an adaptive transmission scheme. Before each communication process, the proposed scheme selects one out of the following transmission modes, under the criterion of maximizing the instantaneous capacity of the secondary network: HD relaying, FD relaying, or DT with no cooperation. In contrast to the common assumption of a coverage-extension scenario, whereby the direct link is considered to be unavailable or to convey a weak interference signal (as in the case of FD relaying), owing to the pathloss

\footnotetext{
${ }^{2}$ Two joint-decoding schemes for relaying networks are regular encoding/sliding-window decoding and regular encoding/backward decoding, whose information-theoretical basis has been developed in [37] and [38], respectively. Those schemes have been generalized in [39] and more recently applied to practical full-duplex relaying scenarios in [23] and [24].
} 
or the severe shadowing caused by large obstacles (see, e.g., [21], [22], [25]-[27], [32]-[34]), our study is focused on exploiting the spatial diversity offered by the direct-link transmission, using it as a source of useful information. ${ }^{3}$ Accordingly, when the CRN operates in HD relaying mode, we consider that the secondary destination merges the received signals coming from the secondary source and relay by employing maximal-ratio combining (MRC), whereas in the FD relaying mode the secondary destination combines these signals by employing JD.

This paper investigates the outage performance of a underlay CRN consisted of one source, one DF relay, and one destination, which employs the proposed adaptive transmission scheme. To the best of our knowledge, this is the first study addressing an adaptive transmission scheme that includes the HD relaying, FD relaying, and DT modes, in the context of an underlay spectrum-sharing scenario. The following are our main contributions:

- Exact closed-form expressions are derived for the joint probability density function (PDF) and cumulative distribution function (CDF) of the instantaneous received signal-to-noise ratios (SNRs) corresponding to the first hop and direct link, and of those corresponding to the second hop and self-interference link at the secondary network. From these statistics, the corresponding expressions for the marginal CDF and PDF are also derived. It is worthwhile to point out that the random variables (RVs) corresponding to the instantaneous received SNRs of the first hop and direct link are indeed correlated, as will be specified later. The same holds true for those corresponding to the second hop and self-interference link. As far as we are aware, this correlation has been generally neglected in the literature, concerning the analysis of CRNs. Moreover, although an analysis derived from the assumption of independence between these RVs may render a good approximation for the outage probability, as in the case of the schemes in [34]-[36], which consider the FD relaying mode only, such assumption leads to a non-negligible gap in performance when an adaptive transmission scheme is considered, as shall be seen in Section VII.

- An exact integral-form expression is derived for the outage probability of the proposed adaptive transmission scheme, which is validated by Monte Carlo simulations. Numerical results reveal a floor in the outage curves at high SNR, after the maximum transmit system power exceeds the maximum interference power constraint, provided that these quantities are varied independently of each other.

- A closed-form approximation is proposed for the aforementioned exact expression, proving to be very tight along the whole range of values of transmit system SNR.

- An asymptotic closed-form expression is obtained for the proposed approximation, so as to characterize the outage

\footnotetext{
${ }^{3}$ The motivation for this is that, in general, the channel power gain of the direct link may be non-negligible. Moreover, even in such adverse fading scenarios, the direct link is likely to be temporarily usable, although it is supposed to be blocked on average [21].
}

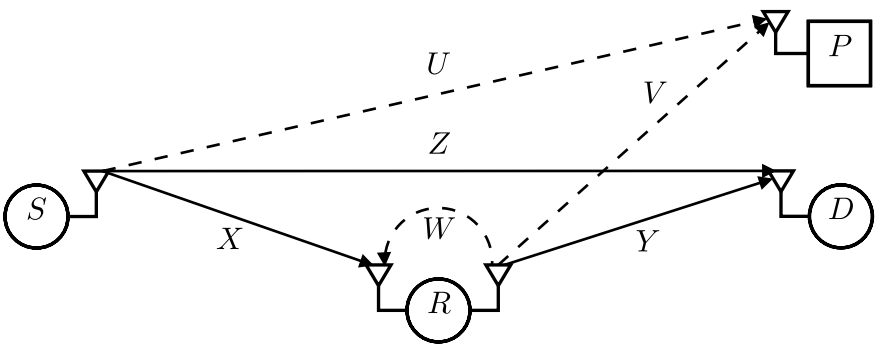

Fig. 1. System model (desired-signal links: solid lines; interference links: dashed lines).

floor at the high-SNR regime and assess the impact of the interference power constraint at the primary receiver on the secondary network.

The remainder of this paper is outlined as follows. Section II details the system model and the proposed adaptive transmission scheme. Section IV characterizes the outage performance of the proposed scheme by deriving an exact integral-form expression. In Section V, we propose a highly accurate closedform approximation for the derived exact outage probability. In Section VI, based on this approximate expression, we characterize the asymptotic outage performance at high SNR. Section VII includes some numerical results and discussions, as well as Monte Carlo simulations to validate our theoretical expressions. Finally, Section VIII provides the main conclusions of the paper. To enhance the flow of the paper, we postpone the proofs for the theorems and other statements to the Appendices A to E.

Throughout this paper, $f_{A}(\cdot)$ and $F_{A}(\cdot)$ respectively denote the PDF and CDF of a generic RV $A, f_{A, B}(\cdot, \cdot)$ and $F_{A, B}(\cdot, \cdot)$ respectively denote the joint PDF and CDF of generic RVs $A$ and $B, E[\cdot]$ denotes expectation, $\operatorname{Pr}[\cdot]$ denotes probability, “ $\approx$ " denotes approximation, and " $\simeq$ " denotes asymptotic equivalence.

\section{SySTEM MODEL}

Consider an underlay CRN composed of one secondary source $S$, one secondary DF relay $R$, and one secondary destination $D$, operating in the presence of a primary receiver $P$, as illustrated in Fig. 1. All nodes are single-antenna devices, except for the secondary relay, which is equipped with one receive antenna and one transmit antenna. ${ }^{4}$ The solid lines denote the desired-signal links, and the dashed lines denote the interference links. Let (i) $h_{X}, h_{Y}$, and $h_{Z}$ respectively denote the channel coefficients of the desired-signal links from $S$ to $R$ (first hop), from $R$ to $D$ (second hop), and from $S$ to $D$ (direct link), at the secondary network; (ii) $h_{W}$ denote the channel coefficient of the $\mathrm{RSI}^{5}$ at $R$,

\footnotetext{
${ }^{4}$ Both FD and HD relaying modes can benefit from using two antennas at the relay, by enabling high physical isolation between the antennas in order to mitigate the self-interference in the former case, and by optimizing the antenna configurations (e.g., placement and directivity) in two different directions in the latter [21].

${ }^{5}$ As usually considered for FD relaying (see, e.g., [21]-[24], [34]-[36]), we model the RSI at the secondary relay as a Rayleigh fading channel, by assuming that the link between its transmit and receive antennas is dominated by the scattering component, while the specular component is considerably attenuated after employing passive and active self-interference mitigation techniques [40].
} 
when FD relaying is the selected mode; and (iii) $h_{U}$ and $h_{V}$ respectively denote the channel coefficients of the interference links from $S$ to $P$ and from $R$ to $P$. All these links are assumed to undergo independent and arbitrarily distributed (i.a.d.) Rayleigh block fading, ${ }^{6}$ so that the channel coefficient $h_{A}$, with $A \in\{X, Y, Z, U, V, W\}$, can be modeled as a zero-mean, independent, circularly symmetric complex Gaussian RV with variance $\Omega_{A}=E\left[\left|h_{A}\right|^{2}\right]$. Correspondingly, the channel power gain $g_{A}=\left|h_{A}\right|^{2}$ follows an exponential distribution with mean value $\Omega_{A}$. Also assume that the received signals at each node are perturbed by additive white Gaussian noise with mean power $N_{0}$. In addition, given an underlay spectrum-sharing scenario, the transmit powers at the secondary source and relay can be written, respectively, as

$$
\begin{aligned}
& P_{S}=\min \left\{\frac{I}{g_{U}}, P_{\mathrm{T}}\right\}= \begin{cases}P_{\mathrm{T}} & \text { if } g_{U} \leq I / P_{\mathrm{T}} \\
I / g_{U} & \text { otherwise, }\end{cases} \\
& P_{R}=\min \left\{\frac{I}{g_{V}}, P_{\mathrm{T}}\right\}= \begin{cases}P_{\mathrm{T}} & \text { if } g_{V} \leq I / P_{\mathrm{T}} \\
I / g_{V} & \text { otherwise, }\end{cases}
\end{aligned}
$$

where $I$ is the maximum tolerable interference power (a.k.a. interference temperature $)^{7}$ caused by $S$ and $R$ at $P$, and $P_{\mathrm{T}}$ is the maximum transmit power at $S$ and $R$. Based on all this, the instantaneous received SNRs of the first hop, second hop, direct link, and self-interference link can be respectively written as $X=g_{X} P_{S} / N_{0}, Y=g_{Y} P_{R} / N_{0}, Z=g_{Z} P_{S} / N_{0}$, and $W=g_{W} P_{R} / N_{0}$, where $P_{S}$ and $P_{R}$ are respectively given by the expressions in (1) and (2). In fact, these expressions are valid only for the HD relaying and DT modes. In the particular case of the FD relaying mode, $P_{S}$ and $P_{R}$ are halved. This is because, for the sake of fairness, we consider that the maximum transmit system power is the same for all the transmission modes, equal to $P_{\mathrm{T}}$. More specifically, during each channel use, provided that the interference power constraint is satisfied, the transmit powers at $S$ and $R$ are set as follows: (i) in the HD relaying mode, we use $P_{S}$ and $P_{R}$, respectively, so that either $S$ or $R$ is allowed to transmit alternately with a maximum power $P_{\mathrm{T}}$; (ii) in the FD relaying mode, we use $P_{S} / 2$ and $P_{R} / 2$, respectively, so that $S$ and $R$ are each allowed to transmit concurrently with a maximum power $P_{\mathrm{T}} / 2$; and (iii) in the DT mode, we use $P_{S}$ at $S$, while dispensing with $R$, so that $S$ is allowed to transmit with maximum power $P_{\mathrm{T}}$. Thus, the following constraints concerning each transmission mode are met:

$$
\begin{array}{rlrl}
g_{U} P_{S} \leq I \text { and } g_{V} P_{R} & \leq I, & \text { for HD relaying; } \\
g_{U} \frac{P_{S}}{2}+g_{V} \frac{P_{R}}{2} & \leq I, & & \text { for FD relaying; } \\
g_{U} P_{S} & \leq I, & & \text { for DT. }
\end{array}
$$

According to the proposed adaptive transmission scheme, the secondary network operates by dynamically switching

\footnotetext{
${ }^{6}$ Herein, all channel coefficients are assumed to remain constant during each data transmission block, but vary independently from one block to another.

${ }^{7}$ As pointed out in [4], regulatory agencies would be responsible for setting an interference-temperature limit, bearing in mind the condition of the radiofrequency environment that exists in the frequency band under consideration. For more details on how the interference temperature is defined, we also refer the reader to [5].
}

among the three considered transmission modes, based on the instantaneous channel conditions. More specifically, before each communication process, the proposed scheme selects the transmission mode that maximizes the instantaneous capacity of the secondary network, that is

$$
\max \left\{C_{\mathrm{FD}}, C_{\mathrm{HD}}, C_{\mathrm{DT}}\right\},
$$

where $C_{\mathrm{FD}}, C_{\mathrm{HD}}$, and $C_{\mathrm{DT}}$ are the instantaneous capacities for the FD relaying, HD relaying, and DT modes, which can be respectively expressed as

$$
\begin{aligned}
& C_{\mathrm{FD}}=\log _{2}\left(1+\min \left\{\frac{X / 2}{1+W / 2}, \frac{Y}{2}+\frac{Z}{2}\right\}\right), \\
& C_{\mathrm{HD}}=\frac{1}{2} \log _{2}(1+\min \{X, Y+Z\}), \\
& C_{\mathrm{DT}}=\log _{2}(1+Z),
\end{aligned}
$$

where in (5a) and (5b) we have considered that the relay operates under the DF protocol. Note in (5a) that the received SNRs at the secondary relay and destination are halved, since we have considered equal power allocation for the secondary source and relay, so that the interference power constraint in (3b) is satisfied. Also note in (5a) that the direct-link transmission is handled at the secondary destination as an information signal, differently from [34, eqs. (13) and (18)], where it is considered as interference. In addition, note in $(5 b)$ that a pre-log factor of $1 / 2$ appears, since the same SU information is transmitted over two channel uses.

\section{Feedback Overhead}

In this section, we detail some aspects concerning the feedback overhead required by the proposed adaptive transmission scheme. One possible approach is to put the burden of the transmission-mode selection task on the secondary source. Accordingly, the secondary source is supposed to continuously monitor the instantaneous CSI corresponding to the desiredsignal links (i.e., $X, Y$, and $Z$ ) and to the interfering links (i.e., $W, U$, and $V$ ). Then, it selects the transmission mode that provides the maximum instantaneous capacity, based on (4). For this purpose, in a first stage (before each communication process), the secondary source can readily acquire its local CSI, that is, the CSI of $X, Z$, and $U$, by estimating the channel coefficients based on the pilot signaling coming from the secondary relay, the secondary destination, and the primary receiver, respectively ${ }^{8}$ [41], [42]. Using the same approach, the secondary relay can acquire its local CSI, that is, the CSI of $Y$, $W$, and $V .{ }^{9}$ Next, the secondary relay feeds back its local CSI to the secondary source for accomplishing the transmissionmode selection task. For this latter stage, by using quantization

\footnotetext{
${ }^{8}$ Based on the reciprocity property of a wireless channel [43], a secondary node will be able to estimate its local CSI in both directions, that is, to and from its secondary/primary neighboring nodes.

${ }^{9}$ It is also worthwhile to point out that, in the case of the CSI corresponding to the interfering links $U$ and $V$, as an alternative approach to the periodic sensing of pilot signals from the primary receiver, the secondary source and relay are able to obtain the information by direct feedback from the primary receiver [44] or by indirect feedback from a third-party, such as a band manager which mediates between the primary and secondary users [45].
} 
feedback, similarly as in [42, Sec. VII], an overhead of $3 n$ bits is required, where $n$ is the number of quantization bits used to feed back the CSI corresponding to $Y, W$, and $V$. For example, by using $n=4,2^{4}=16$ uniform SNR quantization levels are adopted. Then, a feedback overhead of 12 bits is required. Finally, the secondary source broadcast a 2-bit signaling message (e.g., "00", "01", or "10", as one out of three transmission modes are considered) to inform the secondary relay and destination of the selected transmission mode.

\section{EXACT ANALYSIS}

In the proposed adaptive transmission scheme, an outage event occurs when the instantaneous capacity of the system, corresponding to the transmission mode that provides the maximum instantaneous capacity, namely, FD relaying, HD relaying, or DT, is less than a predefined target spectral efficiency $\mathcal{R}$ (bits/s/Hz). Then, the outage probability for such a scheme can be formulated as in the following theorem.

Theorem 1: The exact outage probability for CRNs operating under the proposed adaptive transmission scheme and spectrum-sharing constraints is formulated as

$$
\begin{aligned}
P_{\text {out }}=\operatorname{Pr}\left[\min \left\{\frac{2 X}{2+W}, Y+Z\right\}<2 \tau_{1},\right. \\
\\
\left.\min \{X, Y+Z\}<\tau_{2}, Z<\tau_{1}\right],
\end{aligned}
$$

where $\tau_{1} \triangleq 2^{\mathcal{R}}-1$ and $\tau_{2} \triangleq 2^{2 \mathcal{R}}-1$ are SNR thresholds corresponding to the target spectral efficiency $\mathcal{R}$, the former being defined for the FD relaying and DT modes and the latter for the HD relaying mode.

Proof: The proof is provided in Appendix A.

The expression for $P_{\text {out }}$ in Theorem 1 can be computed as in Proposition 1, to be presented later on. Before proceeding, we present the following lemma, which will be useful in the proof of this proposition.

Lemma 1: Let $\bar{\gamma}_{P} \triangleq P_{\mathrm{T}} / N_{0}$ be the maximum transmit system SNR available at the secondary source and relay, and $\bar{\gamma}_{I} \triangleq I / N_{0}$ be the maximum tolerable interference-tonoise ratio at the primary receiver, so that the instantaneous received SNRs $X$ and $Z$, defined in Section II, can be rewritten as

$$
X=\min \left\{\frac{\bar{\gamma}_{I}}{g_{U}}, \bar{\gamma}_{P}\right\} g_{X}, \quad \text { and } Z=\min \left\{\frac{\bar{\gamma}_{I}}{g_{U}}, \bar{\gamma}_{P}\right\} g_{Z} .
$$

Then, the joint CDF and PDF of $X$ and $Z$ are respectively obtained as

$$
\begin{aligned}
F_{X, Z}(x, z) & \\
= & 1-e^{-\frac{x}{\gamma P \Omega_{X}}}-e^{-\frac{z}{\gamma P \Omega_{Z}}}+e^{-\frac{x}{\gamma P \Omega_{X}}-\frac{z}{\gamma P \Omega_{Z}}} \\
& +\frac{x \Omega_{U} e^{-\frac{\bar{\gamma}_{I}}{\bar{\gamma}_{P} \Omega_{U}}-\frac{x}{\bar{\gamma}_{P} \Omega_{X}}}}{\bar{\gamma}_{I} \Omega_{X}+x \Omega_{U}}+\frac{z \Omega_{U} e^{-\frac{\bar{\gamma}_{I}}{\bar{\gamma} \Omega_{U}}-\frac{z}{\bar{\gamma}_{P} \Omega_{Z}}}}{\bar{\gamma}_{I} \Omega_{Z}+z \Omega_{U}} \\
& -\frac{\Omega_{U}\left(x \Omega_{Z}+z \Omega_{X}\right) e^{-\frac{\bar{\gamma}_{I}}{\bar{\gamma}_{P} \Omega_{U}}-\frac{x}{\bar{\gamma}_{P} \Omega_{X}}-\frac{z}{\bar{\gamma}_{P} \Omega_{Z}}}}{\bar{\gamma}_{I} \Omega_{X} \Omega_{Z}+\Omega_{U}\left(x \Omega_{Z}+z \Omega_{X}\right)}
\end{aligned}
$$

$$
\begin{aligned}
& f_{X, Z}(x, z) \\
& =\frac{e^{-\frac{x}{\gamma_{P} \Omega_{X}}-\frac{z}{\gamma_{P} \Omega_{Z}}}}{\bar{\gamma}_{P}^{2} \Omega_{X} \Omega_{Z}} \\
& -\frac{\Omega_{U} e^{-\frac{\bar{\gamma}_{I}}{\gamma_{P} \Omega_{U}}-\frac{x}{\gamma_{P} \Omega_{X}}-\frac{z}{\gamma^{2} \Omega_{Z}}}}{\bar{\gamma}_{P}^{2} \Omega_{X} \Omega_{Z}\left(\bar{\gamma}_{I} \Omega_{X} \Omega_{Z}+x \Omega_{U} \Omega_{Z}+\Omega_{U} \Omega_{X} z\right)^{3}} \\
& \times\left[\Omega_{U}^{2}\left(x \Omega_{Z}+z \Omega_{X}\right)^{3}+4 \bar{\gamma}_{I} x z \Omega_{U} \Omega_{X}^{2} \Omega_{Z}^{2}\right. \\
& \quad-2 \bar{\gamma}_{I}^{2} \bar{\gamma}_{P} \Omega_{X}^{3} \Omega_{Z}^{3}-2 \bar{\gamma}_{I} \bar{\gamma}_{P}^{2} \Omega_{U} \Omega_{X}^{3} \Omega_{Z}^{3} \\
& \quad+2 \bar{\gamma}_{I} x^{2} \Omega_{U} \Omega_{X} \Omega_{Z}^{3}+2 \bar{\gamma}_{I} z^{2} \Omega_{U} \Omega_{X}^{3} \Omega_{Z} \\
& \quad-2 \bar{\gamma}_{I} \bar{\gamma}_{P} x \Omega_{U} \Omega_{X}^{2} \Omega_{Z}^{3}-2 \bar{\gamma}_{I} \bar{\gamma}_{P} \Omega_{U} \Omega_{X}^{3} \Omega_{Z}^{2} \\
& \left.\quad+\bar{\gamma}_{I}^{2} x \Omega_{X}^{2} \Omega_{Z}^{3}+\bar{\gamma}_{I}^{2} z \Omega_{X}^{3} \Omega_{Z}^{2}\right] .
\end{aligned}
$$

Proof: The proof is provided in Appendix B.

Correspondingly, by rewriting $W$ and $Y$, defined in Section II, as

$$
W=\min \left\{\frac{\bar{\gamma}_{I}}{g_{V}}, \bar{\gamma}_{P}\right\} g_{W}, \quad \text { and } Y=\min \left\{\frac{\bar{\gamma}_{I}}{g_{V}}, \bar{\gamma}_{P}\right\} g_{Y},
$$

it is possible to show from Lemma 1 that their joint CDF and PDF, $F_{W, Y}(\cdot, \cdot)$ and $f_{W, Y}(\cdot, \cdot)$, are respectively given by (8) and (9), with $\Omega_{X}, \Omega_{Z}$, and $\Omega_{U}$ being replaced by $\Omega_{W}, \Omega_{Y}$, and $\Omega_{V}$, in this order. Moreover, using [46, eqs. (6.9) and (6.10)], the marginal CDF and PDF of $X$ can be respectively derived as

$$
\begin{aligned}
F_{X}(x)= & F_{X, Z}(x, \infty) \\
= & 1-e^{-\frac{x}{\bar{\gamma}_{P} \Omega_{X}}}\left(1-\frac{x \Omega_{U} e^{-\frac{\bar{\gamma}_{I}}{\gamma_{P} \Omega_{U}}}}{\bar{\gamma}_{I} \Omega_{X}+x \Omega_{U}}\right), \\
f_{X}(x)= & \int_{0}^{\infty} f_{X, Z}(x, z) d z \\
= & \frac{e^{-\frac{x}{\bar{\gamma}_{P} \Omega_{X}}}}{\bar{\gamma}_{P} \Omega_{X}}\left[1-\Omega_{U} e^{-\frac{\bar{\gamma}_{I}}{\gamma_{P} \Omega_{U}}}\right. \\
& \left.\times \frac{x^{2} \Omega_{U}+x \bar{\gamma}_{I} \Omega_{X}-\bar{\gamma}_{I} \bar{\gamma}_{P} \Omega_{X}^{2}}{\left(\bar{\gamma}_{I} \Omega_{X}+x \Omega_{U}\right)^{2}}\right] .
\end{aligned}
$$

Following the same rationale, the marginal CDF and PDF corresponding to $Z, W$, and $Y$ can be obtained as in (11) and (12), respectively, with the following replacements: $\Omega_{X}$ by $\Omega_{Z}$, for $Z ; \Omega_{X}$ and $\Omega_{U}$ by $\Omega_{W}$ and $\Omega_{V}$, for $W$; and $\Omega_{X}$ and $\Omega_{U}$ by $\Omega_{Y}$ and $\Omega_{V}$, for $Y$.

Remark 1: It should be stressed that the correlation between the SNRs of the first hop and direct link, $X$ and $Z$, as well as between the SNRs of the second hop and self-interference link, $Y$ and $W$, has been neglected in the literature, concerning the analysis of CRN, particularly under the FD relaying mode. As aforementioned, even though an analysis derived from the assumption of independence between these variates may provide a good approximation to the exact outage probability, by considering schemes operating under a given fixed transmission mode only (e.g., as in [34]-[36]), it renders a nonnegligible gap in performance when an adaptive transmission scheme is contemplated, as shall be seen later. In addition, by bearing in mind the intricacy of the joint distributions 
in (8) and (9), it is not surprising that an exact mathematical analysis may become intractable, depending on the complexity of the system under investigation.

Now, with the results of Lemma 1 as our primary tool, we formulate the following proposition on the outage probability of the proposed scheme.

Proposition 1: The exact outage probability for underlay CRNs operating under the proposed adaptive transmission scheme, formulated as in (6), can be solved as in (13), shown at the bottom of this page. In this expression, $\Theta_{1}(w, y)$ and $\Theta_{2}(w, y)$ can be respectively computed as in (14) and (15), also shown at the bottom of this page. In addition, the integral terms defined as $I_{1}$ and $I_{2}$ can be obtained in closed form: the former being solved as in (44), where $\Theta_{3}\left(\tau_{1}\right) \triangleq \int_{0}^{\tau_{1}} F_{X}\left(\tau_{1}(w+2)\right) f_{W}(w) d w$ can be computed in closed form as in (45); and the latter being solved as

$$
I_{2}=\Theta_{4}\left(2 \tau_{1}, 2 \tau_{1}\right)-\Theta_{4}\left(\tau_{1}, 2 \tau_{1}\right),
$$

where $\Theta_{4}(a, b) \triangleq \int_{0}^{a} F_{Z}(b-y) f_{Y}(y) d y$, with $a \leq b$, can also be computed in closed form as in (46). For clarity and ease of reference, the expressions corresponding to $I_{1}, \Theta_{3}\left(\tau_{1}\right)$, and $\Theta_{4}(a, b)$ are shown at the end of the paper. In those expressions, $\operatorname{Ei}(\cdot)$ is the exponential integral function [47, eq. (8.211.1)]. Note in (13) that other integral terms have been defined, as $I_{3}$ to $I_{7}$. Those terms cannot be solved in an exact closed form, but shall be addressed in the next section in an approximate fashion.

Proof: The proof is provided in Appendix C.

Remark 2: Note from (6) that an exact closed-form expression for the outage probability of the proposed transmission scheme proves to be extremely intricate, leading to a four-fold integral-form solution, given as in (31), in Appendix C. Although numerical integration using standard computing softwares such as Mathematica and Matlab is feasible, it is time-consuming, undergoing sometimes slow-convergence issues that may lead to inaccurate results. In turn, in Proposition 1, the original formulation for $P_{\text {out }}$ in (31), composed of five four-fold integral-form terms, was reduced to a significantly simpler, less complex integral-form expression, given as in (13). It should be emphasized that this expression is exact, and, importantly, the single- and two-fold integral terms therein can indeed be efficiently evaluated by numerical methods found in the aforementioned computing softwares. Nevertheless, although our formulation is exact, an alternative closed-form solution for the integral terms in (13) is particularly appealing. In the following section, we propose an approximation to the outage probability, which is solved in closed form. Furthermore, as shall be seen in Section VII, this approximation proves to be highly accurate over the whole range of values of transmit system SNR, for any values of the average RSI channel power at the secondary relay and the interference power constraint at the primary receiver.

\section{Approximate Solution}

In this section, we present a tight closed-form approximation to the exact outage probability of the proposed scheme, given by (13). More specifically, we propose an approximate solution for the integral terms $I_{i}, i=3,4, \ldots, 7$, in the referred expression, as formulated in the following theorem.

$$
\begin{aligned}
& P_{\text {out }}=F_{Y}\left(\tau_{1}\right) F_{Z}\left(\tau_{1}\right)+F_{X, Z}\left(\tau_{2}, \tau_{1}\right)\left[1-F_{W}\left(\tau_{1}\right)-F_{Y}\left(\tau_{2}-\tau_{1}\right)+F_{W, Y}\left(\tau_{1}, \tau_{2}-\tau_{1}\right)\right] \\
& +\underbrace{\int_{0}^{\tau_{1}} F_{X, Z}\left(\tau_{1}(w+2), \tau_{1}\right) f_{W}(w) d w}_{I_{1}}+\underbrace{\int_{\tau_{1}}^{2 \tau_{1}} F_{Z}\left(2 \tau_{1}-y\right) f_{Y}(y) d y}_{I_{2}}-\underbrace{\int_{0}^{\infty} F_{X, Z}\left(\tau_{1}(w+2), \tau_{1}\right) \Theta_{1}\left(w, \tau_{1}\right) d w}_{I_{3}} \\
& +\underbrace{\int_{\tau_{1}}^{\infty} F_{X, Z}\left(\tau_{1}(w+2), \tau_{1}\right) \Theta_{1}\left(w, \tau_{2}-\tau_{1}\right) d w}_{I_{4}}-\underbrace{\int_{\tau_{2}-\tau_{1}}^{\tau_{2}} F_{X, Z}\left(\tau_{2}, \tau_{2}-y\right) \Theta_{2}\left(\tau_{1}, y\right) d y}_{I_{5}} \\
& -\underbrace{\int_{0}^{\infty} \int_{\tau_{1}}^{2 \tau_{1}} F_{X, Z}\left(\tau_{1}(w+2), 2 \tau_{1}-y\right) f_{W, Y}(w, y) d y d w}_{I_{6}}+\underbrace{\int_{\tau_{1}}^{\infty} \int_{\tau_{2}-\tau_{1}}^{\tau_{2}} F_{X, Z}\left(\tau_{1}(w+2), \tau_{2}-y\right) f_{W, Y}(w, y) d y d w}_{I_{7}} \\
& \Theta_{1}(w, y)=\frac{e^{-\frac{w}{\gamma_{P} \Omega_{W}}}}{\bar{\gamma}_{P} \Omega_{W}}-\frac{e^{-\frac{w}{\gamma_{P} \Omega_{W}}-\frac{y}{\gamma_{P} \Omega_{Y}}}}{\bar{\gamma}_{P} \Omega_{W}}-\frac{e^{-\frac{\bar{\gamma}_{I}}{\gamma_{P} \Omega_{V}}-\frac{w}{\gamma_{P} \Omega_{W}}} \Omega_{V}\left(w^{2} \Omega_{V}+w \bar{\gamma}_{I} \Omega_{W}-\bar{\gamma}_{I} \bar{\gamma}_{P} \Omega_{W}^{2}\right)}{\bar{\gamma}_{P} \Omega_{W}\left(\bar{\gamma}_{I} \Omega_{W}+w \Omega_{V}\right)^{2}} \\
& +\frac{e^{-\frac{\bar{\gamma}_{I}}{\bar{\gamma}_{P} \Omega_{V}}-\frac{w}{\bar{\gamma}_{P} \Omega_{W}}-\frac{y}{\bar{\gamma}_{P} \Omega_{Y}}} \Omega_{V}\left[\Omega_{Y}^{2}\left(\bar{\gamma}_{I} \bar{\gamma}_{P} \Omega_{W}^{2}+w \bar{\gamma}_{I} \Omega_{W}+w^{2} \Omega_{V}\right)+y \Omega_{W} \Omega_{Y}\left(\bar{\gamma}_{I} \Omega_{W}+2 w \Omega_{V}\right)+y^{2} \Omega_{V} \Omega_{W}^{2}\right]}{\bar{\gamma}_{P} \Omega_{W}\left(\bar{\gamma}_{I} \Omega_{W} \Omega_{Y}+w \Omega_{V} \Omega_{Y}+y \Omega_{V} \Omega_{W}\right)^{2}} \\
& \Theta_{2}(w, y)=-\frac{e^{-\frac{\bar{\gamma}_{I}}{\gamma_{P} \Omega_{V}}-\frac{y}{\gamma_{P} \Omega_{Y}}-\frac{w}{\gamma_{P} \Omega_{W}}} \Omega_{V}\left[\Omega_{W}^{2}\left(\bar{\gamma}_{I} \bar{\gamma}_{P} \Omega_{Y}^{2}+y \bar{\gamma}_{I} \Omega_{Y}+y^{2} \Omega_{V}\right)+w \Omega_{Y} \Omega_{W}\left(\bar{\gamma}_{I} \Omega_{Y}+2 y \Omega_{V}\right)+w^{2} \Omega_{V} \Omega_{Y}^{2}\right]}{\bar{\gamma}_{P} \Omega_{Y}\left(\bar{\gamma}_{I} \Omega_{Y} \Omega_{W}+w \Omega_{V} \Omega_{Y}+y \Omega_{V} \Omega_{W}\right)^{2}} \\
& +\frac{e^{-\frac{y}{\gamma_{P} \Omega_{Y}}-\frac{w}{\gamma_{P} \Omega_{W}}}}{\bar{\gamma}_{P} \Omega_{Y}}
\end{aligned}
$$


Theorem 2: A closed-form approximation to the outage probability in (13), for underlay CRNs employing the proposed adaptive transmission scheme, is given as

$$
\begin{aligned}
P_{\text {out }} \approx & I_{1}+\left[1-\Theta_{5}\left(\tau_{1}\right)\right]\left[I_{2}+F_{Y}\left(\tau_{1}\right) F_{Z}\left(\tau_{1}\right)\right]+F_{X, Z}\left(\tau_{2}, \tau_{1}\right) \\
& \times\left[1-F_{W}\left(\tau_{1}\right)-F_{Y}\left(\tau_{2}-\tau_{1}\right)+F_{W, Y}\left(\tau_{1}, \tau_{2}-\tau_{1}\right)\right] \\
& +F_{Y}\left(\tau_{2}-\tau_{1}\right) F_{Z}\left(\tau_{1}\right)\left[\Theta_{5}\left(\tau_{1}\right)-\Theta_{3}\left(\tau_{1}\right)\right] \\
& -\left[\Theta_{4}\left(\tau_{2}, \tau_{2}\right)-\Theta_{4}\left(\tau_{2}-\tau_{1}, \tau_{2}\right)\right] \\
& \times\left\{\Theta_{5}\left(\tau_{1}\right)-\Theta_{3}\left(\tau_{1}\right)-\left[1-F_{W}\left(\tau_{1}\right)\right] \times F_{X}\left(\tau_{2}\right)\right\},
\end{aligned}
$$

where $\Theta_{5}\left(\tau_{1}\right)$ can be evaluated as in (47).

Proof: The proof is provided in Appendix D.

\section{AsYMPTOTIC ANALYSIS}

In this section, an asymptotic closed-form expression at the high-SNR regime for the approximation proposed in (17) is presented as follows.

Theorem 3: The asymptotic outage probability at high SNR for underlay CRNs operating under the proposed adaptive transmission scheme is given as

$$
\begin{aligned}
P_{\text {out }} \simeq & \tilde{I}_{1}+\left[1-\tilde{\Theta}_{5}\left(\tau_{1}\right)\right]\left[\tilde{I}_{2}+\tilde{F}_{Y}\left(\tau_{1}\right) \tilde{F}_{Z}\left(\tau_{1}\right)\right]+\tilde{F}_{X, Z}\left(\tau_{2}, \tau_{1}\right) \\
& \times\left[1-\tilde{F}_{W}\left(\tau_{1}\right)-\tilde{F}_{Y}\left(\tau_{2}-\tau_{1}\right)+\tilde{F}_{W, Y}\left(\tau_{1}, \tau_{2}-\tau_{1}\right)\right] \\
& +\tilde{F}_{Y}\left(\tau_{2}-\tau_{1}\right) \tilde{F}_{Z}\left(\tau_{1}\right)\left[\tilde{\Theta}_{5}\left(\tau_{1}\right)-\tilde{\Theta}_{3}\left(\tau_{1}\right)\right] \\
& -\left[\tilde{\Theta}_{4}\left(\tau_{2}, \tau_{2}\right)-\tilde{\Theta}_{4}\left(\tau_{2}-\tau_{1}, \tau_{2}\right)\right] \\
& \times\left\{\tilde{\Theta}_{5}\left(\tau_{1}\right)-\tilde{\Theta}_{3}\left(\tau_{1}\right)-\left[1-\tilde{F}_{W}\left(\tau_{1}\right)\right] \times \tilde{F}_{X}\left(\tau_{2}\right)\right\},
\end{aligned}
$$

where $\tilde{I}_{1}, \tilde{I}_{2}, \tilde{\Theta}_{3}(\cdot), \tilde{\Theta}_{4}(\cdot, \cdot), \tilde{\Theta}_{5}(\cdot), \tilde{F}_{X, Z}(\cdot, \cdot), \tilde{F}_{W, Y}(\cdot, \cdot)$, and $\tilde{F}_{A}(\cdot)$, with $A \in\{W, X, Y, Z\}$, are asymptotic expressions for the corresponding terms in (17), with $\tilde{I}_{1}$ and $\tilde{\Theta}_{3}(\cdot)$ being given by (24) and (25), shown at the bottom of this page, and the rest of terms being given by

$$
\begin{aligned}
\tilde{\Theta}_{4}(a, b) & \frac{\bar{\gamma}_{I} \Omega_{U} \Omega_{V} \Omega_{Y}}{\left(\Omega_{V}\left(b \Omega_{U}+\bar{\gamma}_{I} \Omega_{Z}\right)+\bar{\gamma}_{I} \Omega_{U} \Omega_{Y}\right)^{2}} \\
& \times\left[\frac{a\left(b \Omega_{V}+\bar{\gamma}_{I} \Omega_{Y}\right)\left(\Omega_{V}\left(b \Omega_{U}+\bar{\gamma}_{I} \Omega_{Z}\right)+\bar{\gamma}_{I} \Omega_{U} \Omega_{Y}\right)}{\bar{\gamma}_{I} \Omega_{Y}\left(a \Omega_{V}+\bar{\gamma}_{I} \Omega_{Y}\right)}\right. \\
& \left.\quad+\bar{\gamma}_{I} \Omega_{Z} \log \left(\frac{\bar{\gamma}_{I} \Omega_{Y}\left(\Omega_{U}(b-a)+\bar{\gamma}_{I} \Omega_{Z}\right)}{\left(a \Omega_{V}+\bar{\gamma}_{I} \Omega_{Y}\right)\left(b \Omega_{U}+\bar{\gamma}_{I} \Omega_{Z}\right)}\right)\right],
\end{aligned}
$$

$$
\begin{aligned}
\tilde{\Theta}_{5}\left(\tau_{1}\right) & \frac{\tau_{1} \Omega_{U}}{\left(\tau_{1} \Omega_{U}\left(2 \Omega_{V}-\bar{\gamma}_{I} \Omega_{W}\right)+\bar{\gamma}_{I} \Omega_{V} \Omega_{X}\right)^{2}} \\
& \times\left[\left(2 \Omega_{V}-\bar{\gamma}_{I} \Omega_{W}\right)\left(\tau_{1} \Omega_{U}\left(2 \Omega_{V}-\bar{\gamma}_{I} \Omega_{W}\right)+\bar{\gamma}_{I} \Omega_{V} \Omega_{X}\right)\right. \\
& \left.\quad-\bar{\gamma}_{I}^{2} \Omega_{V} \Omega_{W} \Omega_{X} \log \left(\frac{\bar{\gamma}_{I} \tau_{1} \Omega_{U} \Omega_{W}}{\bar{\gamma}_{I} \Omega_{V} \Omega_{X}+2 \tau_{1} \Omega_{U} \Omega_{V}}\right)\right],
\end{aligned}
$$$$
\tilde{I}_{2}=\tilde{\Theta}_{4}\left(2 \tau_{1}, 2 \tau_{1}\right)-\tilde{\Theta}_{4}\left(\tau_{1}, 2 \tau_{1}\right) \text {, }
$$

$$
\begin{aligned}
& \tilde{F}_{X, Z}(x, z) \\
& =z \Omega_{U}\left(\frac{1}{\bar{\gamma}_{I} \Omega_{Z}+z \Omega_{U}}-\frac{\bar{\gamma}_{I} \Omega_{X}^{2}}{\left(\bar{\gamma}_{I} \Omega_{X}+x \Omega_{U}\right)}\right. \\
& \left.\quad \times \frac{1}{\left(\bar{\gamma}_{I} \Omega_{X} \Omega_{Z}+x \Omega_{U} \Omega_{Z}+z \Omega_{U} \Omega_{X}\right)}\right), \\
& \tilde{F}_{X}(x)=\frac{x \Omega_{U}}{\bar{\gamma}_{I} \Omega_{X}+x \Omega_{U}},
\end{aligned}
$$

$$
\begin{aligned}
& \tilde{I}_{1}=\frac{\tau_{1} \Omega_{U} \Omega_{V}}{\bar{\gamma}_{I} \Omega_{W}+\tau_{1} \Omega_{V}} \\
& \times\left\{\frac{\bar{\gamma}_{I} \Omega_{X}}{\left(\tau_{1} \Omega_{U}\left(2 \Omega_{V}-\bar{\gamma}_{I} \Omega_{W}\right)+\bar{\gamma}_{I} \Omega_{V} \Omega_{X}\right)^{2}\left(\tau_{1} \Omega_{U}\left(\Omega_{V}\left(\Omega_{X}+2 \Omega_{Z}\right)-\bar{\gamma}_{I} \Omega_{W} \Omega_{Z}\right)+\bar{\gamma}_{I} \Omega_{V} \Omega_{X} \Omega_{Z}\right)^{2}}\right. \\
& \times\left\{\overline { \gamma } _ { I } \Omega _ { W } ( \overline { \gamma } _ { I } \Omega _ { W } + \tau _ { 1 } \Omega _ { V } ) \left[\Omega_{Z}^{2}\left(\tau_{1} \Omega_{U}\left(2 \Omega_{V}-\bar{\gamma}_{I} \Omega_{W}\right)+\bar{\gamma}_{I} \Omega_{V} \Omega_{X}\right)^{2}\right.\right. \\
& \times \log \left(\frac{\bar{\gamma}_{I} \Omega_{X} \Omega_{Z}+\tau_{1}^{2} \Omega_{U} \Omega_{Z}+\tau_{1} \Omega_{U}\left(\Omega_{X}+2 \Omega_{Z}\right)}{\bar{\gamma}_{I} \Omega_{X} \Omega_{Z}+\tau_{1} \Omega_{U}\left(\Omega_{X}+2 \Omega_{Z}\right)}\right) \\
& +\log \left(\bar{\gamma}_{I} \Omega_{X}+2 \tau_{1} \Omega_{U}\right)\left(\tau_{1} \Omega_{U}\left(-\bar{\gamma}_{I} \Omega_{W} \Omega_{Z}+\Omega_{V} \Omega_{X}+2 \Omega_{V} \Omega_{Z}\right)+\bar{\gamma}_{I} \Omega_{V} \Omega_{X} \Omega_{Z}\right)^{2} \\
& \left.-\log \left(\bar{\gamma}_{I} \Omega_{X}+\tau_{1}\left(\tau_{1}+2\right) \Omega_{U}\right)\left(\tau_{1} \Omega_{U}\left(-\bar{\gamma}_{I} \Omega_{W} \Omega_{Z}+\Omega_{V} \Omega_{X}+2 \Omega_{V} \Omega_{Z}\right)+\bar{\gamma}_{I} \Omega_{V} \Omega_{X} \Omega_{Z}\right)^{2}\right] \\
& -\tau_{1} \Omega_{V}^{2} \Omega_{X}\left(\tau_{1} \Omega_{U}\left(2 \Omega_{V}-\bar{\gamma}_{I} \Omega_{W}\right)+\bar{\gamma}_{I} \Omega_{V} \Omega_{X}\right)\left(\tau_{1} \Omega_{U}\left(\Omega_{V}\left(\Omega_{X}+2 \Omega_{Z}\right)-\bar{\gamma}_{I} \Omega_{W} \Omega_{Z}\right)+\bar{\gamma}_{I} \Omega_{V} \Omega_{X} \Omega_{Z}\right) \\
& -\bar{\gamma}_{I} \tau_{1} \Omega_{U} \Omega_{V} \Omega_{W} \Omega_{X} \log \left(\bar{\gamma}_{I} \Omega_{W}\right)\left(\bar{\gamma}_{I} \Omega_{W}+\tau_{1} \Omega_{V}\right)\left(\tau_{1} \Omega_{U}\left(\Omega_{V}\left(\Omega_{X}+4 \Omega_{Z}\right)-2 \bar{\gamma}_{I} \Omega_{W} \Omega_{Z}\right)+2 \bar{\gamma}_{I} \Omega_{V} \Omega_{X} \Omega_{Z}\right) \\
& +\bar{\gamma}_{I} \tau_{1} \Omega_{U} \Omega_{V} \Omega_{W} \Omega_{X}\left(\bar{\gamma}_{I} \Omega_{W}+\tau_{1} \Omega_{V}\right) \log \left(\bar{\gamma}_{I} \Omega_{W}+\tau_{1} \Omega_{V}\right) \\
& \left.\left.\times\left(\tau_{1} \Omega_{U}\left(\Omega_{V}\left(\Omega_{X}+4 \Omega_{Z}\right)-2 \bar{\gamma}_{I} \Omega_{W} \Omega_{Z}\right)+2 \bar{\gamma}_{I} \Omega_{V} \Omega_{X} \Omega_{Z}\right)\right\}+\frac{\tau_{1}}{\bar{\gamma}_{I} \Omega_{Z}+\tau_{1} \Omega_{U}}\right\} \\
& \tilde{\Theta}_{3}\left(\tau_{1}\right) \simeq \frac{\tau_{1} \Omega_{U} \Omega_{V}}{\left(\bar{\gamma}_{I} \Omega_{W}+\tau_{1} \Omega_{V}\right)\left(\tau_{1} \Omega_{U}\left(2 \Omega_{V}-\bar{\gamma}_{I} \Omega_{W}\right)+\bar{\gamma}_{I} \Omega_{V} \Omega_{X}\right)^{2}} \\
& \times\left[\overline { \gamma } _ { I } ^ { 2 } \Omega _ { W } \Omega _ { X } ( \overline { \gamma } _ { I } \Omega _ { W } + \tau _ { 1 } \Omega _ { V } ) \left(\log \left(\bar{\gamma}_{I} \Omega_{X}+2 \tau_{1} \Omega_{U}\right)-\log \left(\bar{\gamma}_{I} \Omega_{X}+\tau_{1}\left(\tau_{1}+2\right) \Omega_{U}\right)\right.\right. \\
& \left.\left.+\log \left(\bar{\gamma}_{I} \Omega_{W}+\tau_{1} \Omega_{V}\right)-\log \left(\bar{\gamma}_{I} \Omega_{W}\right)\right)+\tau_{1}\left(2 \Omega_{V}-\bar{\gamma}_{I} \Omega_{W}\right)\left(\tau_{1} \Omega_{U}\left(2 \Omega_{V}-\bar{\gamma}_{I} \Omega_{W}\right)+\bar{\gamma}_{I} \Omega_{V} \Omega_{X}\right)\right]
\end{aligned}
$$




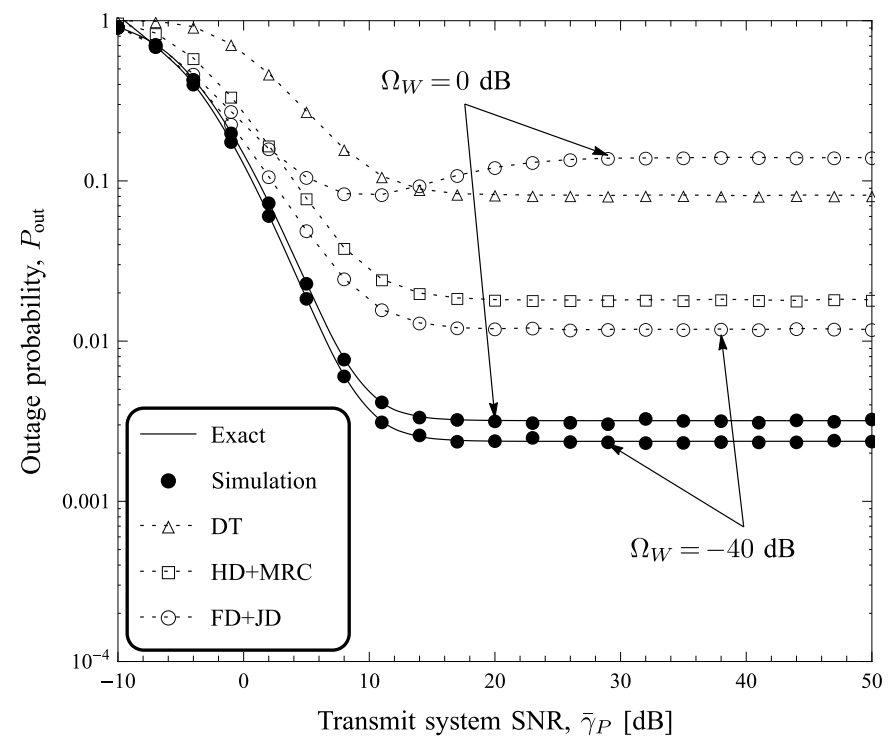

Fig. 2. Outage probability of proposed adaptive transmission scheme versus transmit system SNR, and comparison with fixed transmission schemes, for two different values of average residual self-interference channel power $\Omega_{W}$, with $\bar{\gamma}_{I}=10 \mathrm{~dB}$.

and, after the appropriate substitutions, similarly as in Lemma $1, \tilde{F}_{W, Y}(\cdot, \cdot)$ and $\tilde{F}_{A}(\cdot)$, with $A \in\{W, Y, Z\}$, can be obtained from (22) and (23), respectively.

Proof: The proof is provided in Appendix E.

Remark 3: From (18), we can observe that the asymptotic outage performance does not depend on $\bar{\gamma}_{P}$; instead, it is a constant value given in terms of the following system parameters: (i) average channel powers of the first hop $\left(\Omega_{X}\right)$, second hop $\left(\Omega_{Y}\right)$, direct link $\left(\Omega_{Z}\right)$, self-interference link $\left(\Omega_{W}\right)$, and interference links from the secondary source $\left(\Omega_{U}\right)$ and relay $\left(\Omega_{V}\right)$ on the primary receiver; and (ii) the maximum tolerable interference-to-noise ratio at the primary receiver $\left(\bar{\gamma}_{I}\right)$. Therefore, the diversity order [48] of the considered system is nil. In Section VII, it shall be seen how the asymptotic outage probability is affected by those system parameters.

\section{NUMERICAL RESULTS}

In this section, we assess our new analytical results derived in Sections IV to VI for the outage probability of the proposed adaptive transmission scheme in a number of scenarios of interest. Monte Carlo simulations are also included to corroborate our analysis. For illustration purposes, we consider a 2D plane network with normalized distances, in which the secondary source, relay, and destination are respectively located at $(0,0),(0.5,0)$, and $(1,0)$, and the primary receiver is located at $(0.25,1)$. In addition, we assume that the average channel power between any two nodes is determined by the corresponding distance between them, that is, $\Omega_{A}=d_{A}^{-n}$, with $A \in\{X, Y, Z, U, V\}$, where $d_{A}$ is the distance between the nodes and $n$ is the pathloss exponent. We use $n=4$ and $\mathcal{R}=1 \mathrm{bits} / \mathrm{s} / \mathrm{Hz}$ in our examples.

Fig. 2 shows the outage probability of the proposed adaptive transmission scheme in an underlay spectrum-sharing scenario versus the maximum transmit system SNR, for two different values of average RSI channel power, specifically,
$\Omega_{W}=0,-40 \mathrm{~dB}$. We set $\bar{\gamma}_{I}=10 \mathrm{~dB}$. For comparison purposes, the simulated outage performance for fixed transmission modes (DT, HD relaying with MRC, and FD relaying with JD) operating alone is also provided. For each of the fixed transmission modes, the instantaneous capacity was respectively defined as in (5a)-(5c), in Section II. In addition, for the FD relaying mode, the same aforementioned values of RSI are considered. Equivalence among the different schemes was attained by using the same total transmit power in all cases, as given by (3a)-(3c). Note that our exact analytical expression, given by (13), is validated by the simulation results. Also note that, for all the considered schemes, an outage floor is observed at the high-SNR regime. This is due to the transmit power constraint on the secondary nodes, inherent to the underlay spectrum-access strategy, which is inflicted by the maximum tolerable interference power at the primary receiver. Indeed, we observe that the outage floor is achieved for a transmit system SNR around the value of the interference constraint $\left(\bar{\gamma}_{P} \approx \bar{\gamma}_{I}=10 \mathrm{~dB}\right)$, that is, after the maximum allowed value of transmit SNR is reached. As a result, the outage performance saturates, irrespective of any further increase in the maximum transmit system SNR available at the secondary nodes. On the other hand, we observe from Fig. 2 that, for low SNR values $\left(\bar{\gamma}_{P} \leq 0 \mathrm{~dB}\right)$, the FD relaying mode outperforms the HD relaying mode, whereas for the medium-to-high SNR region, the relative FD performance is determined by the average RSI channel power at the secondary relay. That is, for low values of average RSI channel power (e.g., $\Omega_{W}=-40 \mathrm{~dB}$ ), the FD mode performance always surpasses that attained by the HD mode. By contrast, as the average RSI channel power increases (e.g., $\Omega_{W}=0 \mathrm{~dB}$ ), the outage performance of FD relaying deteriorates, as expected. On the other hand, the proposed adaptive transmission scheme is superior to its fixed HD, FD, and DT counterparts, along the entire SNR region, showcasing that a non-negligible gain can be attained by using a dynamic switching transmission policy. However, also note that in the adaptive transmission scheme, as well as in the FD relaying scheme, as the average RSI channel power increases from -40 to $0 \mathrm{~dB}$, the outage performance deteriorates, as expected, so that the outage floor level also increases. More important, we observe that this performance degradation is less critical in the adaptive transmission case when compared to that undergone by the FD relaying scheme.

Fig. 3 shows simulations for the frequency of use of each transmission mode composing the proposed scheme (i.e., HD, FD, and DT), corresponding to the above-considered scenarios. We can observe that FD relaying is the most frequently selected mode at low SNR, while, interestingly, HD relaying is the less frequently selected mode along the entire SNR region. On the other hand, for medium-to-high SNR, note that the frequency of use of each transmission mode heavily depends on the value of $\Omega_{W}$. More specifically, the lower the value of $\Omega_{W}$, the more frequently selected is the FD relaying mode, as the effects of RSI diminish, while the HD relaying mode is barely selected. On the contrary, as the average RSI channel power increases (i.e., as the deleterious effects of RSI prevails), DT becomes the preferred 


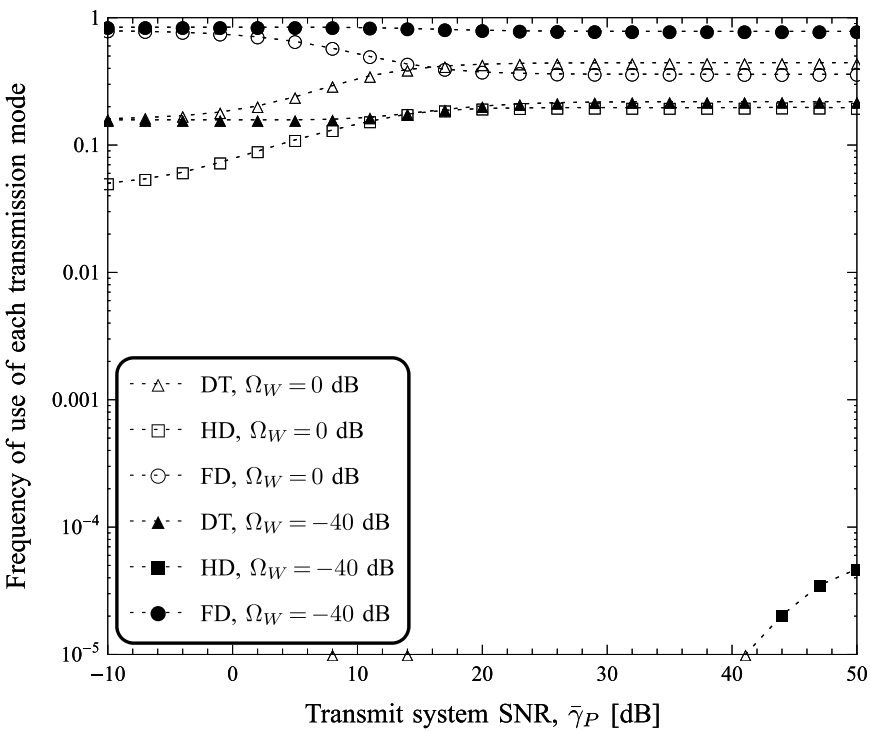

Fig. 3. Frequency of use of each transmission mode versus transmit system SNR for two different values of average residual self-interference channel power $\Omega_{W}$, with $\bar{\gamma}_{I}=10 \mathrm{~dB}$.

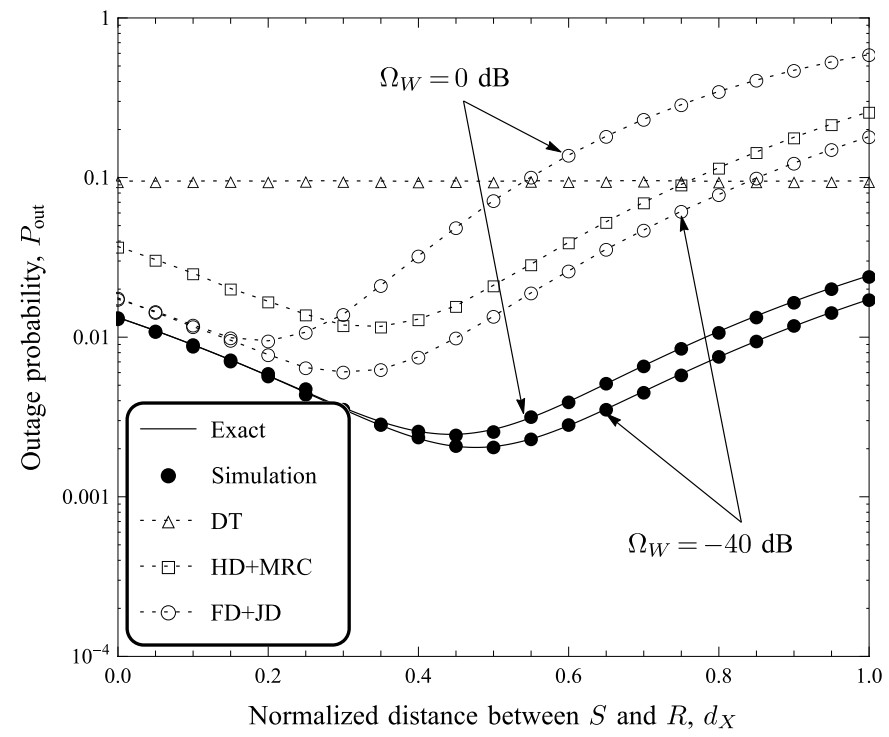

Fig. 4. Outage probability of proposed adaptive transmission scheme versus distance between secondary source and relay, and comparison with fixed transmission schemes, for two different values of average residual selfinterference channel power $\Omega_{W}$, with $\bar{\gamma}_{P}=10 \mathrm{~dB}$ and $\bar{\gamma}_{I}=20 \mathrm{~dB}$.

transmission mode, while HD relaying presents a significant increase in its frequency of use. Other numerical examples not included in this paper show that, for the high-SNR region, the HD relaying mode is more frequently selected than the FD relaying mode (but still less frequently selected than DT) as $\bar{\gamma}_{I}$ increases. This is due to the fact that, as more power is allowed to the secondary relay, the FD relaying performance worsens, and, consequently, the HD relaying mode is more frequently selected.

Fig. 4 shows the outage probability of the proposed adaptive transmission scheme versus the distance $d_{X}$ between the secondary source and relay, for values of $\Omega_{W}=0,-40 \mathrm{~dB}$. For comparison, the outage performance of DT, HD relaying

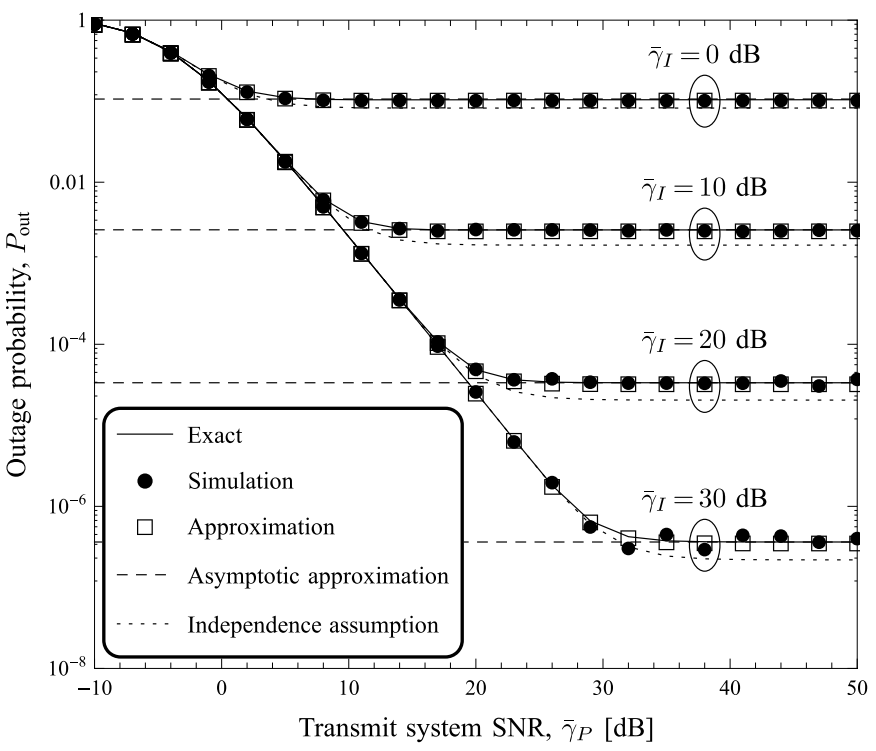

Fig. 5. Outage probability of proposed adaptive transmission scheme versus transmit system SNR for different values of interference constraint $\bar{\gamma}_{I}$, with $\Omega_{W}=-20 \mathrm{~dB}$.

with MRC, and FD relaying with JD, is also illustrated. In this case, the position of $R$ is no longer fixed; instead, it is ranged over the distance between $S$ and $D$, so that $0<d_{X}<1$ and $d_{X}+d_{Y}=1$. In addition, to keep symmetry w.r.t. $S$ and $R$, the primary receiver is located at $\left(d_{X} / 2,1\right)$. We set $\bar{\gamma}_{P}=10 \mathrm{~dB}$ and $\bar{\gamma}_{I}=20 \mathrm{~dB}$. Here again, we observe the superiority of our scheme in terms of outage performance, irrespective of the position of the secondary relay. In particular, the optimal relay position (i.e., the position that minimizes the outage probability) is observed to be roughly midway between $S$ and $D$. In addition, a significant gain with respect to the fixed transmission modes is observed for $d_{X}>0.5$ (i.e., when $R$ is located closer to $D$ ). In general, the performance of both fixed HD and FD relaying schemes (and even of the adaptive transmission scheme, albeit in a less degree) deteriorates as $R$ approaches $D$. This is because the first hop becomes then the bottleneck of the HD and FD relaying schemes, as can be corroborated from (5a) and (5b) in Appendix A. In the particular case of FD relaying, the performance degradation is further aggravated as $\Omega_{W}$ increases. In this sense, note that the proposed adaptive transmission scheme proves to be more robust to the effects of the RSI when compared to the fixed FD relaying mode, whose outage behavior is severely compromised for high values of $\Omega_{W}$ and, particularly, when the relay is far distant from the source.

Fig. 5 shows the impact of the interference power constraint at the primary receiver on the outage performance of the proposed scheme, by considering sample illustrative values of $\bar{\gamma}_{I}=0,10,20,30 \mathrm{~dB}$. As an example, we set $\Omega_{W}=$ $-20 \mathrm{~dB}$. Note how the proposed approximation, given by (17), is extremely tight to the exact outage probability in all the cases, and how the asymptotic expression matches the outage performance in the medium-to-high SNR region. Once again, the numerical examples obtained from all the exact, approximate, and asymptotic derived expressions are supported by 


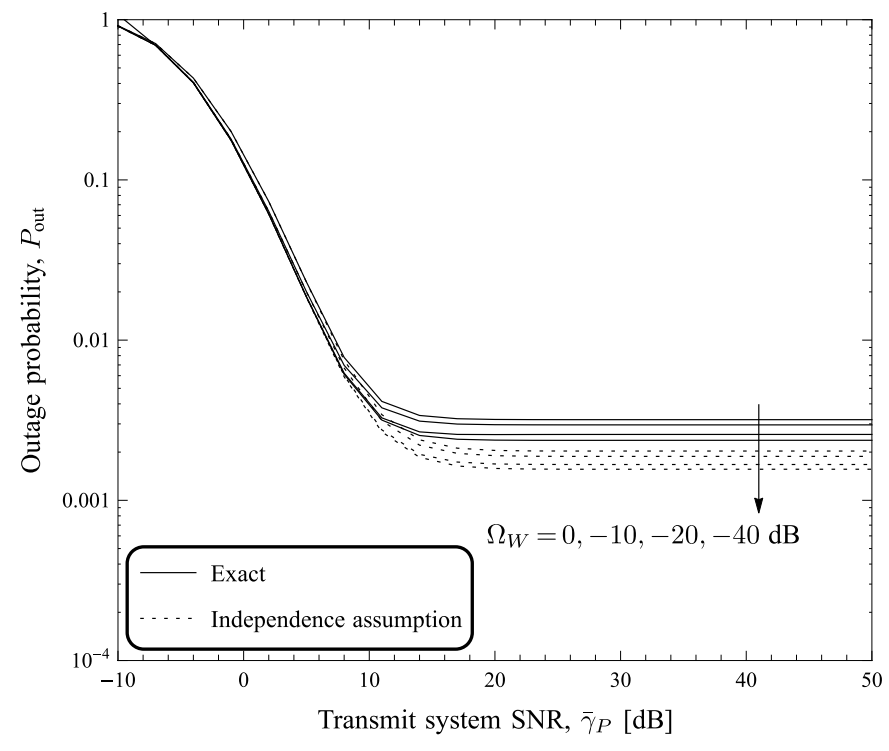

Fig. 6. Comparison between exact and independence-based analysis for the outage probability of the proposed scheme, considering different values of average residual self-interference channel power $\Omega_{W}$, with $\bar{\gamma}_{I}=10 \mathrm{~dB}$.

the simulation results. In the figure, we can observe that, as the maximum tolerable interference power at the primary receiver increases, more transmit power is allowed at the secondary nodes, so that the threshold of transmit system SNR at which the outage floor occurs increases and, therefore, the floor level decreases, thus improving the outage performance. Simulated curves obtained under the assumption of statistical independence between the SNRs $X$ and $Z$, and between $W$ and $Y$ are also shown. Recall from Lemma 1 that $X$ and $Z$, and $W$ and $Y$, are independent pairs of correlated RVs with joint PDF given by (9). Nevertheless, as mentioned before, this correlation has been overlooked in the literature, concerning the performance analysis of CRNs. We can notice from Fig. 5 that an outage analysis based on the statistical-independence assumption between the foregoing RVs leads to results that differ from the actual outage performance, as a non-negligible gap is observed. Fig. 6 corroborates this concern. This figure shows a comparison of the outage probability for the proposed adaptive transmission scheme versus the transmit system SNR between our exact analytical expression, evaluated as in (13), and the simulation results obtained under the independence assumption, ${ }^{10}$ for different values of average RSI channel power $\Omega_{W}=0,-10,-20,-40 \mathrm{~dB}$. As can be seen from the figure, there always exists a performance gap, irrespective of the value of average RSI channel power.

\section{CONCLUSIONS}

This paper presented an adaptive transmission scheme for an underlay cognitive DF relaying network, whereby, before each communication process, one out of the following transmission modes is dynamically selected: HD relaying, FD relaying,

\footnotetext{
${ }^{10}$ Here, for simulation purposes, independence between the instantaneous received SNRs $X$ and $Z$, defined as in (7), is attained by considering independent samples of the channel power gain between the secondary source and the primary receiver, $g_{U}$. A similar assumption is applied to the SNRs $W$ and $Y$ with respect to the channel power gain $g_{V}$.
}

or DT. An exact outage analysis was carried, which showed that the proposed scheme can significantly boost the system performance in comparison with the conventional fixed transmission modes, thus overcoming the limitations inherent to $\mathrm{HD}$ and FD relaying, namely, spectral-efficiency loss and residual self-interference. In addition, an approximate closedform expression was proposed for the exact outage probability of the proposed scheme, which proved to be very tight to the exact solution over the whole range of transmit system SNR. From the latter expression, an asymptotic analysis at the high SNR-regime showed that the outage performance exhibits a floor, provided that the transmit system SNR is varied independently of the interference power constraint at the primary receiver. The floor level was analytically characterized in terms of the system parameters. Numerical results validated our analysis and showcased the accuracy of the proposed approximation.

\section{APPENDIX A \\ PROOF OF THEOREM 1}

An outage occurs if the maximum information rate supported by the system falls below a certain target spectral efficiency $\mathcal{R}$. The corresponding outage event is then given as $\left\{\max \left\{C_{\mathrm{FD}}, C_{\mathrm{HD}}, C_{\mathrm{DT}}\right\}<R\right\}$, where $C_{\mathrm{FD}}, C_{\mathrm{HD}}$, and $C_{\mathrm{DT}}$ are the instantaneous capacities for the FD relaying, HD relaying, and DT modes, given by (5). Hence, the probability of this outage event is obtained as

$$
\begin{aligned}
P_{\mathrm{out}} & =\operatorname{Pr}\left[\max \left\{C_{\mathrm{FD}}, C_{\mathrm{HD}}, C_{\mathrm{DT}}\right\}<\mathcal{R}\right] \\
& =\operatorname{Pr}\left[C_{\mathrm{FD}}<\mathcal{R}, C_{\mathrm{HD}}<\mathcal{R}, C_{\mathrm{DT}}<\mathcal{R}\right] .
\end{aligned}
$$

For a given spectral efficiency $\mathcal{R}$, the target SNR thresholds for the FD relaying and DT modes and for the HD relaying mode are defined as $\tau_{1} \triangleq 2^{\mathcal{R}}-1$ and $\tau_{2} \triangleq 2^{2 \mathcal{R}}-1$, respectively, where, for the HD relaying mode, we have considered that the same SU information is transmitted over two channel uses (i.e., the pre-log factor of $1 / 2$ ). Now, by substituting (5) into (26), and after some algebraic manipulations, the outage probability of the proposed scheme can be rewritten as in (6), which completes the proof.

\section{APPENDIX B \\ PROOF OF LEMMA 1}

By defining $\Lambda \triangleq \min \left\{\bar{\gamma}_{I} / g_{U}, \bar{\gamma}_{P}\right\}$, it follows from the total probability theorem (continuous version) [46, eq. (4.80)] that the joint $\mathrm{CDF}$ of $X$ and $Z$ can be obtained as

$$
F_{X, Z}(x, z)=\int_{0}^{\infty} F_{X, Z \mid \Lambda}(x, z \mid \lambda) f_{\Lambda}(\lambda) d \lambda,
$$

where $F_{X, Z \mid \Lambda}(x, z \mid \lambda)$ can be developed from [46, eq. (6.1)] and (7) as

$$
\begin{aligned}
F_{X, Z \mid \Lambda}(x, z \mid \lambda) & =\operatorname{Pr}[X<x, Z<z \mid \lambda] \\
& =\operatorname{Pr}\left[\lambda g_{X}<x \mid \lambda\right] \operatorname{Pr}\left[\lambda g_{Z}<z \mid \lambda\right] \\
& =F_{g_{X}}\left(\frac{x}{\lambda}\right) F_{g_{Z}}\left(\frac{z}{\lambda}\right) \\
& \stackrel{(a)}{=}\left(1-e^{-\frac{x}{\lambda \Omega_{X}}}\right)\left(1-e^{-\frac{z}{\lambda \Omega_{Z}}}\right),
\end{aligned}
$$




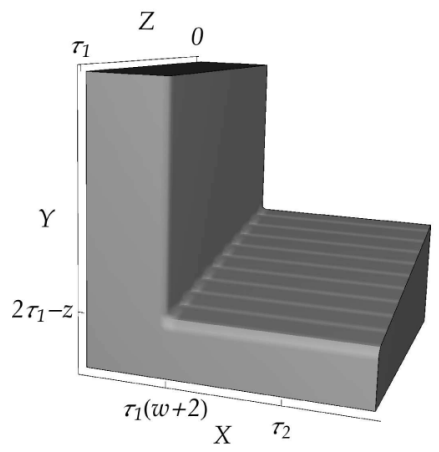

(a)

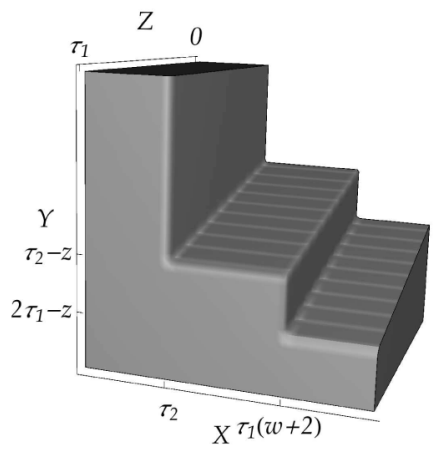

(b)
Fig. 7. Integration regions for $w$ : (a) $0<w<\tau_{1}$; (b) $w>\tau_{1}$.

where in step $(a)$ we have used the fact that $F_{g_{X}}(\cdot)$ and $F_{g_{Z}}(\cdot)$ are the CDFs of exponential RVs with mean values $\Omega_{X}$ and $\Omega_{Z}$, respectively. Note from (27) that the PDF of $\Lambda$ is required in order to determine $F_{X, Z}(x, z)$. To this end, we first derive the $\mathrm{CDF}$ of $\Lambda$, as follows:

$$
\begin{aligned}
F_{\Lambda}(\lambda) & =\operatorname{Pr}\left[\min \left\{\frac{\bar{\gamma}_{I}}{g_{U}}, \bar{\gamma}_{P}\right\}<\lambda\right] \\
& =\operatorname{Pr}\left[\frac{\bar{\gamma}_{I}}{g_{U}}<\lambda, g_{U}>\frac{\bar{\gamma}_{I}}{\bar{\gamma}_{P}}\right]+\operatorname{Pr}\left[\bar{\gamma}_{P}<\lambda, g_{U}<\frac{\bar{\gamma}_{I}}{\bar{\gamma}_{P}}\right] \\
& = \begin{cases}\operatorname{Pr}\left[g_{U}>\bar{\gamma}_{I} / \lambda\right] & \text { if } \lambda \leq \bar{\gamma}_{P} \\
\operatorname{Pr}\left[g_{U}>\bar{\gamma}_{I} / \bar{\gamma}_{P}\right]+\operatorname{Pr}\left[g_{U}<\bar{\gamma}_{I} / \bar{\gamma}_{P}\right] & \text { otherwise }\end{cases} \\
& = \begin{cases}1-F_{g_{U}}\left(\bar{\gamma}_{I} / \lambda\right) & \text { if } \lambda \leq \bar{\gamma}_{P} \\
1 & \text { otherwise, }\end{cases}
\end{aligned}
$$

where $F_{g_{U}}(\cdot)$ is the CDF of an exponential RV with mean value $\Omega_{U}$. Now, by differentiating (29) w.r.t. $\lambda$, the PDF of $\Lambda$ can be obtained as

$$
\begin{aligned}
f_{\Lambda}(\lambda) & =\frac{\bar{\gamma}_{I}}{\lambda^{2}} f_{g_{U}}\left(\frac{\bar{\gamma}_{I}}{\lambda}\right) \mathrm{u}\left(\bar{\gamma}_{P}-\lambda\right)+F_{g_{U}}\left(\frac{\bar{\gamma}_{I}}{\bar{\gamma}_{P}}\right) \delta\left(\lambda-\bar{\gamma}_{P}\right) \\
& =\frac{\bar{\gamma}_{I}}{\lambda^{2} \Omega_{U}} e^{-\frac{\bar{\nu}_{I}}{\lambda \Omega_{U}}} \mathrm{u}\left(\bar{\gamma}_{P}-\lambda\right)+\left(1-e^{-\frac{\bar{\gamma}_{I}}{\gamma_{P} \Omega_{U}}}\right) \delta\left(\lambda-\bar{\gamma}_{P}\right),
\end{aligned}
$$

where $\delta(\cdot)$ denotes the Dirac delta function, $\mathrm{u}(\cdot)$ denotes the unit step function, and $f_{g_{U}}(\cdot)$ is the PDF of an exponential RV with mean value $\Omega_{U}$. Hence, substituting (28) and (30) into (27), and integrating the result over $\lambda$ with the use of the Sifting Property [49], after some algebraic manipulations, we arrive at a new useful expression for the joint CDF of $X$ and $Z$, given as in (8). Finally, using $f_{X, Z}(x, z)=$ $\partial F_{X, Z}(x, z) / \partial x \partial z$ [46, eq. (6.5)], and after lengthy simplifications, a new expression for the joint PDF of $X$ and $Z$ can be obtained as in (9), which concludes the proof.

\section{APPENDIX C \\ PROOF OF PROPOSITION 1}

We notice from (6) that the exact calculation of $P_{\text {out }}$ involves a four-fold integration of the joint PDF of $W, X, Y$, and $Z$ over the region defined by the outage events in the argument of $\operatorname{Pr}[\cdot]$. To do so, we can start the analysis of $P_{\text {out }}$ by partitioning the integration interval of $w$ into two subintervals: (i) $0<w<\left(\tau_{2} / \tau_{1}-2\right)=\tau_{1}$ and (ii) $w>\tau_{1}$, as depicted in Fig. 7. Then, the integration regions determined by the outage events in (6) can be specified as follows. For $0<w<\tau_{1}$, we have $\tau_{1}(w+2)<\tau_{2}$, so that the integration region is given by (Fig. 7a)

$$
\left\{X<\tau_{1}(W+2)\right\} \bigcup\left\{Y+Z<2 \tau_{1}\right\} .
$$

Otherwise, for $w>\tau_{1}$, we have $\tau_{2}<\tau_{1}(w+2)$, so that the integration region is given by (Fig. 7b)

$$
\begin{gathered}
\left\{X<\tau_{2}\right\} \bigcup\left(\left\{\tau_{2}<X<\tau_{1}(W+2)\right\} \bigcap\left\{Y+Z<\tau_{2}\right\}\right) \\
\bigcup\left(\left\{X>\tau_{1}(W+2)\right\} \bigcap\left\{Y+Z<2 \tau_{1}\right\}\right) .
\end{gathered}
$$

Thus, we can develop $P_{\text {out }}$ in the form of a four-fold integral as

$$
\begin{aligned}
P_{\text {out }}= & \int_{0}^{\tau_{1}} \int_{0}^{\tau_{1}} \int_{0}^{\infty} \int_{0}^{\tau_{1}(w+2)} f_{X, Z}(x, z) f_{W, Y}(w, y) \\
& \times d x d y d z d w \\
& +\int_{0}^{\tau_{1}} \int_{0}^{\tau_{1}} \int_{0}^{2 \tau_{1}-z} \int_{\tau_{1}(w+2)}^{\infty} f_{X, Z}(x, z) f_{W, Y}(w, y) \\
& \times d x d y d z d w \\
& +\int_{\tau_{1}}^{\infty} \int_{0}^{\tau_{1}} \int_{0}^{\infty} \int_{0}^{\tau_{2}} f_{X, Z}(x, z) f_{W, Y}(w, y) \\
& \times d x d y d z d w \\
& +\int_{\tau_{1}}^{\infty} \int_{0}^{\tau_{1}} \int_{0}^{\tau_{2}-z} \int_{\tau_{2}}^{\tau_{1}(w+2)} f_{X, Z}(x, z) f_{W, Y}(w, y) \\
& \times d x d y d z d w \\
& +\int_{\tau_{1}}^{\infty} \int_{0}^{\tau_{1}} \int_{0}^{2 \tau_{1}-z} \int_{\tau_{1}(w+2)}^{\infty} f_{X, Z}(x, z) f_{W, Y}(w, y) \\
& \times d x d y d z d w,
\end{aligned}
$$

where we have used $f_{W, X, Y, Z}(w, x, y, z)=f_{X, Z}(x, z) \times$ $f_{W, Y}(w, y)$. This follows from the fact that the instantaneous received SNRs at $R$ and $D$ from $S$, i.e., $X$ and $Z$, both depend on the channel power gain of the interference link from $S$ to $P$, namely, $g_{U}$. Likewise, the instantaneous received SNRs at $R$ and $D$ from $R$, i.e., $W$ and $Y$, both depend on the channel power gain of the interference link from $R$ to $P$, namely, $g_{V}$ (vide definitions for $X$ and $Z$, and for $W$ and $Y$, in Section IV, given in (7) and (10), respectively). Thus, $X$ and $Z$, and $W$ and $Y$ constitute independent pairs of correlated RVs with joint PDFs given by (9), with $\bar{\gamma}_{P}$ and $\bar{\gamma}_{I}$ being defined as in Lemma 1. Now, from (31), using joint-statistics properties [46, eqs. (6.6) and (6.10)] in the first and third terms, and combining the second and fifth terms, we obtain

$$
\begin{aligned}
P_{\text {out }}= & \overbrace{\int_{0}^{\tau_{1}} F_{X, Z}\left(\tau_{1}(w+2), \tau_{1}\right) f_{W}(w) d w}^{P_{\text {out }, 1}} \\
& +\overbrace{\int_{0}^{\infty} \int_{0}^{\tau_{1}} \int_{0}^{2 \tau_{1}-z} \int_{\tau_{1}(w+2)}^{\infty} f_{X, Z}(x, z) f_{W, Y}(w, y)}^{P_{\text {out } 2}} \\
& \times d x d y d z d w \\
& +\overbrace{F_{X, Z}\left(\tau_{2}, \tau_{1}\right)\left[1-F_{W}\left(\tau_{1}\right)\right]}^{P_{\text {out }, 3}} \\
& +\overbrace{\int_{\tau_{1}}^{\infty} \int_{0}^{\tau_{1}} \int_{0}^{\tau_{2}-z} \int_{\tau_{2}}^{\tau_{1}(w+2)} f_{X, Z}(x, z) f_{W, Y}(w, y)} \\
& \times d x d y d z d w,
\end{aligned}
$$




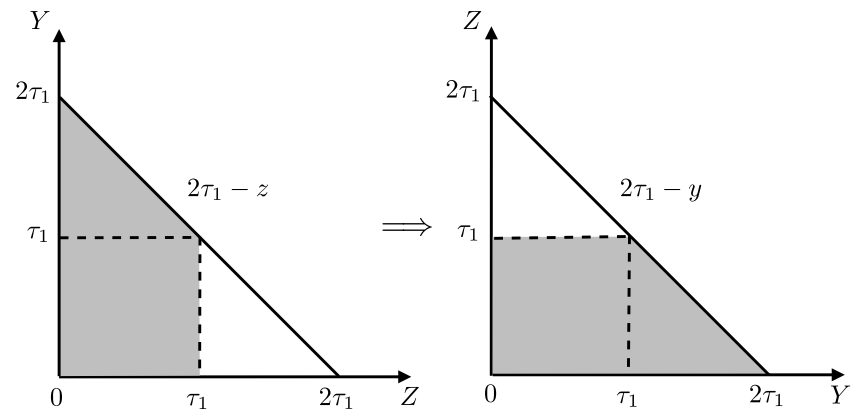

Fig. 8. Change of the order of integration between $Y$ and $Z$ for the term $P_{\text {out }, 2}$ in (32).

where $P_{\text {out, } 2}$ can be solved as in (33), shown at the bottom of this page, as follows. In step $(b)$, we have changed the order of integration between $y$ and $z$ [47, Sec. 4.61], as depicted in Fig. 8. In step (c), we have rewritten the integration interval of $x \in\left[\tau_{1}(w+2), \infty\right)$ as $x \in[0, \infty) \backslash\left[0, \tau_{1}(w+2)\right]$, thus splitting each four-fold integral term in step $(b)$ into two new terms. Finally, in step $(d)$, we have used jointstatistics properties [46, eqs. (6.6) and (6.9)]. In addition, $P_{\text {out, } 4}$ in (32) can be computed as in (34), shown at the bottom of this page, after using a similar approach as in the derivation of $P_{\text {out }, 2}$.
In the foregoing expressions, with the use of [46, eq. (6.11)], $\Theta_{1}(w, y)$ and $\Theta_{2}(w, y)$ can be computed in closed form as

$$
\begin{aligned}
\Theta_{1}(w, y) & =\frac{\partial F_{W, Y}(w, y)}{\partial w}, \\
\Theta_{2}(w, y) & =\int_{0}^{\infty} f_{W, Y}(\lambda, y) d \lambda-\int_{0}^{w} f_{W, Y}(\lambda, y) d \lambda \\
& =f_{Y}(y)-\frac{\partial F_{W, Y}(w, y)}{\partial y},
\end{aligned}
$$

where $f_{Y}(\cdot)$ is given by (12), with $\Omega_{X}$ and $\Omega_{U}$ being replaced by $\Omega_{Y}$ and $\Omega_{V}$, respectively. Then, after some lengthy algebraic manipulations, (35) and (36) can be solved as in (14) and (15). Finally, by summarizing these results, the outage probability in (6) is evaluated as $P_{\text {out }}=\sum_{i=1}^{4} P_{\text {out }, i}$, given as in (13), which completes the proof.

\section{APPENDIX D}

PROOF OF THE THEOREM 2

By assuming statistical independence between the first hop and the direct link, and between the second hop and the self-interference link, we have $f_{X, Z}(x, z)=f_{X}(x) f_{Z}(z)$ and $f_{W, Y}(w, y)=f_{W}(w) f_{Y}(y)$. As aforementioned, this assumption provides an accurate approximation to the integral terms in (13). Then, it follows from the definitions of

$$
\begin{aligned}
& P_{\text {out }, 2} \stackrel{(b)}{=} \int_{0}^{\infty} \int_{0}^{\tau_{1}} \int_{0}^{\tau_{1}} \int_{\tau_{1}(w+2)}^{\infty} f_{X, Z}(x, z) f_{W, Y}(w, y) d x d z d y d w+\int_{0}^{\infty} \int_{\tau_{1}}^{2 \tau_{1}} \int_{0}^{2 \tau_{1}-y} \int_{\tau_{1}(w+2)}^{\infty} f_{X, Z}(x, z) f_{W, Y}(w, y) d x d z d y d w \\
& \stackrel{(c)}{=} \int_{0}^{\infty} \int_{0}^{\tau_{1}} \int_{0}^{\tau_{1}} \int_{0}^{\infty} f_{X, Z}(x, z) f_{W, Y}(w, y) d x d z d y d w-\int_{0}^{\infty} \int_{0}^{\tau_{1}} \int_{0}^{\tau_{1}} \int_{0}^{\tau_{1}(w+2)} f_{X, Z}(x, z) f_{W, Y}(w, y) d x d z d y d w \\
& +\int_{0}^{\infty} \int_{\tau_{1}}^{2 \tau_{1}} \int_{0}^{2 \tau_{1}-y} \int_{0}^{\infty} f_{X, Z}(x, z) f_{W, Y}(w, y) d x d z d y d w-\int_{0}^{\infty} \int_{\tau_{1}}^{2 \tau_{1}} \int_{0}^{2 \tau_{1}-y} \int_{0}^{\tau_{1}(w+2)} f_{X, Z}(x, z) f_{W, Y}(w, y) d x d z d y d w \\
& \stackrel{(d)}{=} F_{Z}\left(\tau_{1}\right) F_{Y}\left(\tau_{1}\right)-\int_{0}^{\infty} F_{X, Z}\left(\tau_{1}(w+2), \tau_{1}\right) \underbrace{\int_{0}}_{\int_{0}^{\tau_{1}} f_{W, Y}(w, y) d y} d w+\int_{\tau_{1}}^{2 \tau_{1}} F_{Z}\left(2 \tau_{1}-y\right) f_{Y}(y) d y \\
& -\int_{0}^{\infty} \int_{\tau_{1}}^{2 \tau_{1}} F_{X, Z}\left(\tau_{1}(w+2), 2 \tau_{1}-y\right) f_{W, Y}(w, y) d y d w \\
& P_{\text {out }, 4}=\int_{\tau_{1}}^{\infty} \int_{0}^{\tau_{2}-\tau_{1}} \int_{0}^{\tau_{1}} \int_{\tau_{2}}^{\tau_{1}(w+2)} f_{X, Z}(x, z) f_{W, Y}(w, y) d x d z d y d w \\
& +\int_{\tau_{1}}^{\infty} \int_{\tau_{2}-\tau_{1}}^{\tau_{2}} \int_{0}^{\tau_{2}-y} \int_{\tau_{2}}^{\tau_{1}(w+2)} f_{X, Z}(x, z) f_{W, Y}(w, y) d x d z d y d w \\
& =\int_{\tau_{1}}^{\infty} \int_{0}^{\tau_{2}-\tau_{1}}\left[F_{X, Z}\left(\tau_{1}(w+2), \tau_{1}\right)-F_{X, Z}\left(\tau_{2}, \tau_{1}\right)\right] f_{W, Y}(w, y) d y d w \\
& +\int_{\tau_{1}}^{\infty} \int_{\tau_{2}-\tau_{1}}^{\tau_{2}}\left[F_{X, Z}\left(\tau_{1}(w+2), \tau_{2}-y\right)-F_{X, Z}\left(\tau_{2}, \tau_{2}-y\right)\right] \\
& \times f_{W, Y}(w, y) d y d w \\
& =\int_{\tau_{1}}^{\infty} F_{X, Z}\left(\tau_{1}(w+2), \tau_{1}\right) \underbrace{\int_{0}^{\tau_{2}-\tau_{1}} f_{W, Y}(w, y) d y}_{\Theta_{1}\left(w, \tau_{2}-\tau_{1}\right)} d w-F_{X, Z}\left(\tau_{2}, \tau_{1}\right)\left[F_{Y}\left(\tau_{2}-\tau_{1}\right)-F_{W, Y}\left(\tau_{1}, \tau_{2}-\tau_{1}\right)\right] \\
& +\int_{\tau_{1}}^{\infty} \int_{\tau_{2}-\tau_{1}}^{\tau_{2}} F_{X, Z}\left(\tau_{1}(w+2), \tau_{2}-y\right) f_{W, Y}(w, y) d y d w-\int_{\tau_{2}-\tau_{1}}^{\tau_{2}} F_{X, Z}\left(\tau_{2}, \tau_{2}-y\right) \underbrace{\int_{\tau_{1}}^{\infty} f_{W, Y}(w, y) d w d y}_{\Theta_{2}\left(\tau_{1}, y\right)}
\end{aligned}
$$


$I_{i}, i=3,4, \ldots, 7$, in $(13)$, and $\Theta_{1}(w, y)$ and $\Theta_{2}(w, y)$ in (33) and (34) that

$$
\begin{aligned}
I_{3} & \approx \int_{0}^{\infty} F_{X}\left(\tau_{1}(w+2)\right) F_{Z}\left(\tau_{1}\right) \int_{0}^{\tau_{1}} f_{W}(w) f_{Y}(y) d y d w \\
& =F_{Y}\left(\tau_{1}\right) F_{Z}\left(\tau_{1}\right) \underbrace{\int_{0}^{\infty} F_{X}\left(\tau_{1}(w+2)\right) f_{W}(w) d w}_{\Theta_{5}\left(\tau_{1}\right)}
\end{aligned}
$$

$$
\begin{aligned}
I_{4} & \approx \int_{\tau_{1}}^{\infty} F_{X}\left(\tau_{1}(w+2)\right) F_{Z}\left(\tau_{1}\right) \int_{0}^{\tau_{2}-\tau_{1}} f_{W}(w) f_{Y}(y) d y d w \\
& =F_{Y}\left(\tau_{2}-\tau_{1}\right) F_{Z}\left(\tau_{1}\right) \underbrace{\int_{\tau_{1}}^{\infty} F_{X}\left(\tau_{1}(w+2)\right) f_{W}(w) d w}_{I_{8}},
\end{aligned}
$$

$$
\begin{aligned}
& I_{1}=\Theta_{3}\left(\tau_{1}\right)-e^{-\frac{\tau_{1}}{\gamma_{P} \Omega_{Z}}} F_{W}\left(\tau_{1}\right)\left(1-\frac{e^{-\frac{\bar{\gamma}_{I}}{\bar{\gamma}_{P} \Omega_{U}}} \tau_{1} \Omega_{U}}{\tau_{1} \Omega_{U}+\bar{\gamma}_{I} \Omega_{Z}}\right)-\frac{e^{-\frac{\bar{\gamma}_{I}}{\bar{\gamma}_{P} \Omega_{V}}-\frac{\tau_{1}}{\gamma_{P} \Omega_{Z}}-\frac{2 \tau_{1}}{\bar{\gamma}_{P} \Omega_{X}}}}{\bar{\gamma}_{P} \Omega_{V}} \\
& \times\left\{\frac{\bar{\gamma}_{P} \tau_{1} \Omega_{V}}{\tau_{1} \Omega_{W}+\Omega_{X}}\left(\frac{e^{-\frac{\tau_{1}\left(\tau_{1} \Omega_{W}+\Omega_{X}\right)}{\gamma_{P} \Omega_{W} \Omega_{X}}}\left(\bar{\gamma}_{I} \Omega_{W}^{2}-\Omega_{V} \Omega_{X}\right)}{\tau_{1} \Omega_{V}+\bar{\gamma}_{I} \Omega_{W}}-\Omega_{W}\right)-\frac{e^{\frac{\bar{\gamma}_{I}\left(\tau_{1} \Omega_{W}+\Omega_{X}\right)}{\gamma_{P} \Omega_{V} \Omega_{X}}} \bar{\gamma}_{I} \tau_{1} \Omega_{W}}{\Omega_{X}}\left[\operatorname{Ei}\left(-\frac{\bar{\gamma}_{I}\left(\tau_{1} \Omega_{W}+\Omega_{X}\right)}{\bar{\gamma}_{P} \Omega_{V} \Omega_{X}}\right)\right.\right. \\
& \left.\left.-\operatorname{Ei}\left(-\frac{\left(\tau_{1} \Omega_{V}+\bar{\gamma}_{I} \Omega_{W}\right)\left(\tau_{1} \Omega_{W}+\Omega_{X}\right)}{\bar{\gamma}_{P} \Omega_{V} \Omega_{W} \Omega_{X}}\right)\right]\right\}+\frac{e^{-\frac{\tau_{1}\left(\Omega_{X}+2 \Omega_{Z}\right)}{\tilde{\gamma}_{P} \Omega_{X} \Omega_{Z}}} \Omega_{X}}{\tau_{1} \Omega_{W}+\Omega_{X}}\left(1-e^{-\frac{\tau_{1}\left(\tau_{1} \Omega_{W}+\Omega_{X}\right)}{\tilde{\gamma}_{P} \Omega_{W} \Omega_{X}}}\right) \\
& +\frac{e^{-\frac{\Omega_{U} \Omega_{W} \Omega_{Z} \tau_{1}^{2}+\Omega_{U}\left(\Omega_{X} \Omega_{Z}+\Omega_{W}\left(\Omega_{X}+2 \Omega_{Z}\right) \tau_{1}+\bar{\gamma}_{I} \Omega_{W} \Omega_{X} \Omega_{Z}\right.}{\tilde{\gamma}_{P} \Omega_{U} \Omega_{W} \Omega_{X} \Omega_{Z}}}}{\bar{\gamma}_{P} \tau_{1} \Omega_{U} \Omega_{W}\left(\tau_{1} \Omega_{W}+\Omega_{X}\right)} \\
& \times\left\{\left(1-e^{\frac{\tau_{1}\left(\tau_{1} \Omega_{W}+\Omega_{X}\right)}{\tilde{\gamma}_{P} \Omega_{W} \Omega_{X}}}\right) \bar{\gamma}_{P} \tau_{1} \Omega_{U} \Omega_{W} \Omega_{X}\right. \\
& -e^{\frac{\left(\tau_{1} \Omega_{W}+\Omega_{X}\right)\left(\Omega_{U} \Omega_{Z} \tau_{1}^{2}+\Omega_{U}\left(\Omega_{X}+2 \Omega_{Z}\right) \tau_{1}+\bar{\gamma}_{I} \Omega_{X} \Omega_{Z}\right)}{\tilde{\gamma}_{P} \tau_{1} \Omega_{U} \Omega_{W} \Omega_{X} \Omega_{Z}}}\left[\operatorname{Ei}\left(-\frac{\left(\tau_{1} \Omega_{W}+\Omega_{X}\right)\left(\bar{\gamma}_{I} \Omega_{X} \Omega_{Z}+\tau_{1} \Omega_{U}\left(\Omega_{X}+2 \Omega_{Z}\right)\right)}{\bar{\gamma}_{P} \tau_{1} \Omega_{U} \Omega_{W} \Omega_{X} \Omega_{Z}}\right)\right. \\
& \left.\left.-\operatorname{Ei}\left(-\frac{\left(\tau_{1} \Omega_{W}+\Omega_{X}\right)\left(\Omega_{U} \Omega_{Z} \tau_{1}^{2}+\Omega_{U}\left(\Omega_{X}+2 \Omega_{Z}\right) \tau_{1}+\bar{\gamma}_{I} \Omega_{X} \Omega_{Z}\right)}{\bar{\gamma}_{P} \tau_{1} \Omega_{U} \Omega_{W} \Omega_{X} \Omega_{Z}}\right)\right] \bar{\gamma}_{I} \Omega_{X}\left(\tau_{1} \Omega_{W}+\Omega_{X}\right)\right\} \\
& e^{-\frac{\bar{\gamma}_{I}\left(\frac{1}{\Omega_{V}}+\frac{1}{\Omega_{U}}\right)+\tau_{1}\left(\frac{\tau_{1}+2}{\Omega_{X}}+\frac{1}{\Omega_{W}}+\frac{1}{\Omega_{Z}}\right)}{\bar{\gamma}_{P}}}
\end{aligned}
$$

$$
\begin{aligned}
& \overline{\bar{\gamma}_{P} \tau_{1} \Omega_{U} \Omega_{V} \Omega_{W}\left(\tau_{1} \Omega_{V}+\bar{\gamma}_{I} \Omega_{W}\right) \Omega_{X}\left(\tau_{1} \Omega_{W}+\Omega_{X}\right)\left(\bar{\gamma}_{I} \Omega_{V} \Omega_{X} \Omega_{Z}+\tau_{1} \Omega_{U}\left(\Omega_{V}\left(\Omega_{X}+2 \Omega_{Z}\right)-\bar{\gamma}_{I} \Omega_{W} \Omega_{Z}\right)\right)^{2}} \\
& \times\left\{\overline { \gamma } _ { P } \tau _ { 1 } \Omega _ { U } \Omega _ { V } \Omega _ { W } \Omega _ { X } [ \overline { \gamma } _ { I } \Omega _ { V } \Omega _ { X } \Omega _ { Z } + \tau _ { 1 } \Omega _ { U } ( \Omega _ { V } ( \Omega _ { X } + 2 \Omega _ { Z } ) - \overline { \gamma } _ { I } \Omega _ { W } \Omega _ { Z } ) ] \left[\Omega_{U} \Omega_{V} \Omega_{X}\left(\Omega_{V} \Omega_{X}-\bar{\gamma}_{I} \Omega_{W}^{2}\right) \tau_{1}^{2}\right.\right. \\
& +\Omega_{Z}\left(\bar{\gamma}_{I} \Omega_{U} \Omega_{W}^{2}\left(\bar{\gamma}_{I} \Omega_{W}-2 \Omega_{V}\right) \tau_{1}^{2}+\Omega_{U} \Omega_{V}\left(2 \Omega_{V}-\bar{\gamma}_{I} \Omega_{W}\right) \Omega_{X} \tau_{1}^{2}+\bar{\gamma}_{I} \Omega_{V}\left(\tau_{1} \Omega_{V}+\bar{\gamma}_{I} \Omega_{W}\right) \Omega_{X}^{2}\right) \\
& \left.+e^{\frac{\tau_{1}\left(\tau_{1} \Omega_{W}+\Omega_{X}\right)}{\tilde{\gamma}_{P} \Omega_{W} \Omega_{X}}}\left(\tau_{1} \Omega_{V}+\bar{\gamma}_{I} \Omega_{W}\right)\left(\tau_{1}^{2} \Omega_{U} \Omega_{W}\left(\Omega_{V} \Omega_{X}+2 \Omega_{V} \Omega_{Z}-\bar{\gamma}_{I} \Omega_{W} \Omega_{Z}\right)-\bar{\gamma}_{I} \Omega_{V} \Omega_{X}^{2} \Omega_{Z}\right)\right] \\
& +\bar{\gamma}_{I}\left(\tau_{1} \Omega_{V}+\bar{\gamma}_{I} \Omega_{W}\right)\left(\tau_{1} \Omega_{W}+\Omega_{X}\right)\left\{e ^ { \frac { ( \tau _ { 1 } \Omega _ { V } + \overline { \gamma } _ { I } \Omega _ { W } ) ( \tau _ { 1 } \Omega _ { W } + \Omega _ { X } ) } { \gamma _ { P } \Omega _ { V } \Omega _ { W } \Omega _ { X } } } \tau _ { 1 } ^ { 2 } \Omega _ { U } ^ { 2 } \Omega _ { W } ^ { 2 } \left[-\bar{\gamma}_{I} \bar{\gamma}_{P} \Omega_{V}^{2} \Omega_{X}^{2} \Omega_{Z}^{2}\right.\right. \\
& \left.+\bar{\gamma}_{I} \tau_{1} \Omega_{V} \Omega_{X} \Omega_{Z}\left(\Omega_{V} \Omega_{X}+2 \Omega_{V} \Omega_{Z}-\bar{\gamma}_{I} \Omega_{W} \Omega_{Z}\right)+\tau_{1}^{2} \Omega_{U}\left(\Omega_{V} \Omega_{X}+2 \Omega_{V} \Omega_{Z}-\bar{\gamma}_{I} \Omega_{W} \Omega_{Z}\right)^{2}\right] \operatorname{Ei}\left(-\frac{\bar{\gamma}_{I}\left(\tau_{1} \Omega_{W}+\Omega_{X}\right)}{\bar{\gamma}_{P} \Omega_{V} \Omega_{X}}\right) \\
& -e^{\frac{\left(\tau_{1} \Omega_{V}+\bar{\gamma}_{I} \Omega_{W}\right)\left(\tau_{1} \Omega_{W}+\Omega_{X}\right)}{\bar{\gamma}_{P} \Omega_{V} \Omega_{W} \Omega_{X}}} \tau_{1}^{2} \Omega_{U}^{2} \Omega_{W}^{2}\left[-\bar{\gamma}_{I} \bar{\gamma}_{P} \Omega_{V}^{2} \Omega_{X}^{2} \Omega_{Z}^{2}+\bar{\gamma}_{I} \tau_{1} \Omega_{V} \Omega_{X} \Omega_{Z}\left(\Omega_{V} \Omega_{X}+2 \Omega_{V} \Omega_{Z}-\bar{\gamma}_{I} \Omega_{W} \Omega_{Z}\right)\right. \\
& \left.+\tau_{1}^{2} \Omega_{U}\left(\Omega_{V} \Omega_{X}+2 \Omega_{V} \Omega_{Z}-\bar{\gamma}_{I} \Omega_{W} \Omega_{Z}\right)^{2}\right] \operatorname{Ei}\left(-\frac{\left(\tau_{1} \Omega_{V}+\bar{\gamma}_{I} \Omega_{W}\right)\left(\tau_{1} \Omega_{W}+\Omega_{X}\right)}{\bar{\gamma}_{P} \Omega_{V} \Omega_{W} \Omega_{X}}\right) \\
& -e^{\frac{\left(\tau_{1} \Omega_{W}+\Omega_{X}\right)\left(\Omega_{U} \Omega_{Z} \tau_{1}^{2}+\Omega_{U}\left(\Omega_{X}+2 \Omega_{Z}\right) \tau_{1}+\bar{\gamma}_{I} \Omega_{X} \Omega_{Z}\right)}{\tilde{\gamma}_{P} \tau_{1} \Omega_{U} \Omega_{W} \Omega_{X} \Omega_{Z}}} \Omega_{V}^{2} \Omega_{X}^{2}\left[\tau_{1}^{2} \Omega_{U}^{2}\left(\Omega_{V}\left(\Omega_{X}+2 \Omega_{Z}\right)^{2}-\bar{\gamma}_{I} \Omega_{W} \Omega_{Z}\left(\Omega_{X}+\bar{\gamma}_{P} \Omega_{W} \Omega_{Z}+2 \Omega_{Z}\right)\right)\right. \\
& \left.+\bar{\gamma}_{I} \tau_{1} \Omega_{X} \Omega_{Z} \Omega_{U}\left(2 \Omega_{V}\left(\Omega_{X}+2 \Omega_{Z}\right)-\bar{\gamma}_{I} \Omega_{W} \Omega_{Z}\right)+\bar{\gamma}_{I}^{2} \Omega_{V} \Omega_{X}^{2} \Omega_{Z}^{2}\right] \\
& \times\left[\operatorname{Ei}\left(-\frac{\left(\tau_{1} \Omega_{W}+\Omega_{X}\right)\left(\bar{\gamma}_{I} \Omega_{X} \Omega_{Z}+\tau_{1} \Omega_{U}\left(\Omega_{X}+2 \Omega_{Z}\right)\right)}{\bar{\gamma}_{P} \tau_{1} \Omega_{U} \Omega_{W} \Omega_{X} \Omega_{Z}}\right)\right. \\
& \left.\left.\left.-\operatorname{Ei}\left(-\frac{\left(\tau_{1} \Omega_{W}+\Omega_{X}\right)\left(\Omega_{U} \Omega_{Z} \tau_{1}^{2}+\Omega_{U}\left(\Omega_{X}+2 \Omega_{Z}\right) \tau_{1}+\bar{\gamma}_{I} \Omega_{X} \Omega_{Z}\right)}{\bar{\gamma}_{P} \tau_{1} \Omega_{U} \Omega_{W} \Omega_{X} \Omega_{Z}}\right)\right]\right\}\right\}
\end{aligned}
$$




$$
\begin{aligned}
I_{5} \approx & \int_{\tau_{2}-\tau_{1}}^{\tau_{2}} F_{X}\left(\tau_{2}\right) F_{Z}\left(\tau_{2}-y\right) \int_{\tau_{1}}^{\infty} f_{W}(w) f_{Y}(y) d w d y \\
= & {\left[1-F_{W}\left(\tau_{1}\right)\right] F_{X}\left(\tau_{2}\right) \underbrace{\int_{\tau_{2}-\tau_{1}}^{\tau_{2}} F_{Z}\left(\tau_{2}-y\right) f_{Y}(y) d y}_{I_{9}} } \\
I_{6} \approx & \underbrace{\int_{0}^{\infty} F_{X}\left(\tau_{1}(w+2)\right) f_{W}(w) d w}_{\Theta_{5}\left(\tau_{1}\right)} \\
& \times \underbrace{\int_{\tau_{1}}^{2 \tau_{1}} F_{Z}\left(2 \tau_{1}-y\right) f_{Y}(y) d y}_{I_{2}}
\end{aligned}
$$

$$
\begin{aligned}
I_{7} \approx & \underbrace{\int_{\tau_{1}}^{\infty} F_{X}\left(\tau_{1}(w+2)\right) f_{W}(w) d w}_{I_{8}} \\
& \times \underbrace{\int_{\tau_{2}-\tau_{1}}^{\tau_{2}} F_{Z}\left(\tau_{2}-y\right) f_{Y}(y) d y}_{I_{9}},
\end{aligned}
$$

where $I_{8}$ and $I_{9}$ can be solved as

$$
\begin{aligned}
& I_{8}=\Theta_{5}\left(\tau_{1}\right)-\Theta_{3}\left(\tau_{1}\right), \\
& I_{9}=\Theta_{4}\left(\tau_{2}, \tau_{2}\right)-\Theta_{4}\left(\tau_{2}-\tau_{1}, \tau_{2}\right),
\end{aligned}
$$

$$
\begin{aligned}
& \Theta_{3}\left(\tau_{1}\right) \triangleq \int_{0}^{\tau_{1}} F_{X}\left(\tau_{1}(w+2)\right) f_{W}(w) d w=\frac{e^{-\frac{2 \tau_{1} \Omega_{V}+\bar{\gamma}_{I} \Omega_{X}}{\gamma_{P} \Omega_{V} \Omega_{X}}}}{\bar{\gamma}_{P} \Omega_{V}} \\
& \times\left\{\frac{\bar{\gamma}_{P} \tau_{1} \Omega_{V}}{\tau_{1} \Omega_{W}+\Omega_{X}}\left(\frac{e^{-\frac{\tau_{1}\left(\tau_{1} \Omega_{W}+\Omega_{X}\right)}{\bar{\gamma}_{P} \Omega_{W} \Omega_{X}}}\left(\bar{\gamma}_{I} \Omega_{W}^{2}-\Omega_{V} \Omega_{X}\right)}{\tau_{1} \Omega_{V}+\bar{\gamma}_{I} \Omega_{W}}-\Omega_{W}\right)\right. \\
& \left.-\frac{e^{\frac{\bar{\gamma}_{I}\left(\tau_{1} \Omega_{W}+\Omega_{X}\right)}{\tilde{\gamma}_{P} \Omega_{V} \Omega_{X}}} \bar{\gamma}_{I} \tau_{1} \Omega_{W}}{\Omega_{X}}\left[\operatorname{Ei}\left(-\frac{\bar{\gamma}_{I}\left(\tau_{1} \Omega_{W}+\Omega_{X}\right)}{\bar{\gamma}_{P} \Omega_{V} \Omega_{X}}\right)-\operatorname{Ei}\left(-\frac{\left(\tau_{1} \Omega_{V}+\bar{\gamma}_{I} \Omega_{W}\right)\left(\tau_{1} \Omega_{W}+\Omega_{X}\right)}{\bar{\gamma}_{P} \Omega_{V} \Omega_{W} \Omega_{X}}\right)\right]\right\} \\
& +\frac{e^{-\frac{\Omega_{U} \Omega_{W} \tau_{1}^{2}+\Omega_{U}\left(4 \Omega_{W}+\Omega_{X}\right) \tau_{1}+\bar{\gamma}_{I} \Omega_{W} \Omega_{X}}{\gamma_{P} \Omega_{U} \Omega_{W} \Omega_{X}}} \Omega_{X}}{\bar{\gamma}_{P} \tau_{1} \Omega_{U} \Omega_{W}\left(\tau_{1} \Omega_{W}+\Omega_{X}\right)} \\
& \times\left\{e^{\frac{\left(\tau_{1}+4\right) \Omega_{U} \Omega_{W} \tau_{1}^{2}+\left(\left(\tau_{1}+2\right) \Omega_{U}+\bar{\gamma}_{I} \Omega_{W}\right) \Omega_{X} \tau_{1}+\bar{\gamma}_{I} \Omega_{X}^{2}}{\bar{\gamma}_{P} \tau_{1} \Omega_{U} \Omega_{W} \Omega_{X}}} \bar{\gamma}_{I}\left(\tau_{1} \Omega_{W}+\Omega_{X}\right)\right. \\
& \times \operatorname{Ei}\left(-\frac{\left(\tau_{1} \Omega_{W}+\Omega_{X}\right)\left(2 \tau_{1} \Omega_{U}+\bar{\gamma}_{I} \Omega_{X}\right)}{\bar{\gamma}_{P} \tau_{1} \Omega_{U} \Omega_{W} \Omega_{X}}\right)+e^{\frac{2 \tau_{1}}{\gamma_{P} \Omega_{X}}}\left[\left(-1+e^{\frac{\tau_{1}\left(\tau_{1} \Omega_{W}+\Omega_{X}\right)}{\bar{\gamma}_{P} \Omega_{W} \Omega_{X}}}\right) \bar{\gamma}_{P} \tau_{1} \Omega_{U} \Omega_{W}\right. \\
& \left.\left.-e^{\frac{\left(\tau_{1} \Omega_{W}+\Omega_{X}\right)\left(\tau_{1}\left(\tau_{1}+2\right) \Omega_{U}+\bar{\gamma}_{I} \Omega_{X}\right)}{\gamma_{P} \tau_{1} \Omega_{U} \Omega_{W} \Omega_{X}}} \bar{\gamma}_{I}\left(\tau_{1} \Omega_{W}+\Omega_{X}\right) \operatorname{Ei}\left(-\frac{\left(\tau_{1} \Omega_{W}+\Omega_{X}\right)\left(\tau_{1}\left(\tau_{1}+2\right) \Omega_{U}+\bar{\gamma}_{I} \Omega_{X}\right)}{\bar{\gamma}_{P} \tau_{1} \Omega_{U} \Omega_{W} \Omega_{X}}\right)\right]\right\} \\
& +F_{W}\left(\tau_{1}\right)+\frac{e^{-\frac{2 \tau_{1}}{\gamma \Omega_{X}}} \Omega_{X}}{\tau_{1} \Omega_{W}+\Omega_{X}}\left(-1+e^{-\frac{\tau_{1}\left(\tau_{1} \Omega_{W}+\Omega_{X}\right)}{\tilde{\gamma}_{P} \Omega_{W} \Omega_{X}}}\right) \\
& e^{-\frac{\bar{\gamma}_{I}}{\gamma_{P}}\left(\frac{1}{\Omega_{V}}+\frac{1}{\Omega_{U}}\right)-\frac{\tau_{1}}{\gamma_{P}}\left(\frac{\tau_{1}+2}{\Omega_{X}}+\frac{1}{\Omega_{W}}\right)} \\
& \overline{\bar{\gamma}_{P} \tau_{1} \Omega_{U} \Omega_{V} \Omega_{W}\left(\tau_{1} \Omega_{V}+\bar{\gamma}_{I} \Omega_{W}\right) \Omega_{X}\left(\tau_{1} \Omega_{U}\left(2 \Omega_{V}-\bar{\gamma}_{I} \Omega_{W}\right)+\bar{\gamma}_{I} \Omega_{V} \Omega_{X}\right)^{2}} \\
& \times\left\{\overline { \gamma } _ { I } ( \tau _ { 1 } \Omega _ { V } + \overline { \gamma } _ { I } \Omega _ { W } ) \left\{e ^ { \frac { ( \tau _ { 1 } \Omega _ { W } + \Omega _ { X } ) ( \tau _ { 1 } ( \tau _ { 1 } + 2 ) \Omega _ { U } + \overline { \gamma } _ { I } \Omega _ { X } ) } { \overline { \gamma } _ { P } \tau _ { 1 } \Omega _ { U } \Omega _ { W } \Omega _ { X } } } \Omega _ { V } ^ { 2 } \Omega _ { X } ^ { 2 } \left(\tau_{1}^{2}\left(4 \Omega_{V}-\bar{\gamma}_{I} \Omega_{W}\left(\bar{\gamma}_{P} \Omega_{W}+2\right)\right) \Omega_{U}^{2}\right.\right.\right. \\
& \left.+\bar{\gamma}_{I} \tau_{1}\left(4 \Omega_{V}-\bar{\gamma}_{I} \Omega_{W}\right) \Omega_{X} \Omega_{U}+\bar{\gamma}_{I}^{2} \Omega_{V} \Omega_{X}^{2}\right) \\
& \times\left[\operatorname{Ei}\left(-\frac{\left(\tau_{1} \Omega_{W}+\Omega_{X}\right)\left(2 \tau_{1} \Omega_{U}+\bar{\gamma}_{I} \Omega_{X}\right)}{\bar{\gamma}_{P} \tau_{1} \Omega_{U} \Omega_{W} \Omega_{X}}\right)-\operatorname{Ei}\left(-\frac{\left(\tau_{1} \Omega_{W}+\Omega_{X}\right)\left(\tau_{1}\left(\tau_{1}+2\right) \Omega_{U}+\bar{\gamma}_{I} \Omega_{X}\right)}{\bar{\gamma}_{P} \tau_{1} \Omega_{U} \Omega_{W} \Omega_{X}}\right)\right] \\
& -e^{\frac{\left(\tau_{1} \Omega_{V}+\bar{\gamma}_{I} \Omega_{W}\right)\left(\tau_{1} \Omega_{W}+\Omega_{X}\right)}{\bar{\gamma}_{P} \Omega_{V} \Omega_{W} \Omega_{X}}} \tau_{1}^{2} \Omega_{U}^{2} \Omega_{W}^{2}\left(\tau_{1}^{2} \Omega_{U}\left(\bar{\gamma}_{I} \Omega_{W}-2 \Omega_{V}\right)^{2}-\bar{\gamma}_{I} \bar{\gamma}_{P} \Omega_{V}^{2} \Omega_{X}^{2}+\bar{\gamma}_{I} \tau_{1} \Omega_{V}\left(2 \Omega_{V}-\bar{\gamma}_{I} \Omega_{W}\right) \Omega_{X}\right) \\
& \left.\times\left[\operatorname{Ei}\left(-\frac{\bar{\gamma}_{I}\left(\tau_{1} \Omega_{W}+\Omega_{X}\right)}{\bar{\gamma}_{P} \Omega_{V} \Omega_{X}}\right)-\operatorname{Ei}\left(-\frac{\left(\tau_{1} \Omega_{V}+\bar{\gamma}_{I} \Omega_{W}\right)\left(\tau_{1} \Omega_{W}+\Omega_{X}\right)}{\bar{\gamma}_{P} \Omega_{V} \Omega_{W} \Omega_{X}}\right)\right]\right\} \\
& -\frac{\bar{\gamma}_{P} \tau_{1} \Omega_{U} \Omega_{V} \Omega_{W} \Omega_{X}\left(\tau_{1} \Omega_{U}\left(\bar{\gamma}_{I} \Omega_{W}-2 \Omega_{V}\right)-\bar{\gamma}_{I} \Omega_{V} \Omega_{X}\right)}{\tau_{1} \Omega_{W}+\Omega_{X}} \\
& \times\left\{\bar{\gamma}_{I} \Omega_{U} \Omega_{W}^{2}\left(2 \Omega_{V}-\bar{\gamma}_{I} \Omega_{W}\right) \tau_{1}^{2}+\Omega_{U} \Omega_{V}\left(\bar{\gamma}_{I} \Omega_{W}-2 \Omega_{V}\right) \Omega_{X} \tau_{1}^{2}\right. \\
& \left.\left.-\bar{\gamma}_{I} \Omega_{V}\left(\tau_{1} \Omega_{V}+\bar{\gamma}_{I} \Omega_{W}\right) \Omega_{X}^{2}+e^{\frac{\tau_{1}\left(\tau_{1} \Omega_{W}+\Omega_{X}\right)}{\bar{\gamma}_{P} \Omega_{W} \Omega_{X}}}\left(\tau_{1} \Omega_{V}+\bar{\gamma}_{I} \Omega_{W}\right)\left(\Omega_{U} \Omega_{W}\left(\bar{\gamma}_{I} \Omega_{W}-2 \Omega_{V}\right) \tau_{1}^{2}+\bar{\gamma}_{I} \Omega_{V} \Omega_{X}^{2}\right)\right\}\right\}
\end{aligned}
$$




$$
\begin{aligned}
& \Theta_{4}(a, b) \triangleq \int_{0}^{a} F_{Z}(b-y) f_{Y}(y) d y=1-e^{-\frac{a}{\gamma P \Omega_{Y}}}\left(1-\frac{a \Omega_{V} e^{-\frac{\bar{\gamma}_{I}}{\gamma_{P} \Omega_{V}}}}{a \Omega_{V}+\bar{\gamma}_{I} \Omega_{Y}}\right)-\frac{e^{-\frac{b}{\gamma_{P} \Omega_{Z}}-\frac{\bar{\gamma}_{I}}{\gamma_{P} \Omega_{U}}-\frac{\bar{\gamma}_{I}}{\gamma_{P} \Omega_{V}}}}{\bar{\gamma}_{P} \Omega_{U} \Omega_{V} \Omega_{Y}\left(b \Omega_{U} \Omega_{V}+\bar{\gamma}_{I} \Omega_{U} \Omega_{Y}+\bar{\gamma}_{I} \Omega_{V} \Omega_{Z}\right)^{2}} \\
& \times\left\{\frac { \overline { \gamma } _ { I } } { \Omega _ { Z } } \left\{\Omega_{V}^{2} \Omega_{Z}^{2} e^{\frac{\left(\Omega_{Y}-\Omega_{Z}\right)\left(b \Omega_{U}+\bar{\gamma}_{I} \Omega_{Z}\right)}{\bar{\gamma}_{P} \Omega_{U} \Omega_{Y} \Omega_{Z}}}\left[\Omega_{U}^{2}\left(b^{2} \Omega_{V}+b \bar{\gamma}_{I} \Omega_{Y}-\bar{\gamma}_{I} \bar{\gamma}_{P} \Omega_{Y}^{2}\right)+\bar{\gamma}_{I} \Omega_{U} \Omega_{Z}\left(2 b \Omega_{V}+\bar{\gamma}_{I} \Omega_{Y}\right)+\bar{\gamma}_{I}^{2} \Omega_{V} \Omega_{Z}^{2}\right]\right.\right. \\
& \times\left[\operatorname{Ei}\left(\frac{\left(\Omega_{Y}-\Omega_{Z}\right)\left(a \Omega_{U}-b \Omega_{U}-\bar{\gamma}_{I} \Omega_{Z}\right)}{\bar{\gamma}_{P} \Omega_{U} \Omega_{Y} \Omega_{Z}}\right)-\operatorname{Ei}\left(-\frac{\left(\Omega_{Y}-\Omega_{Z}\right)\left(b \Omega_{U}+\bar{\gamma}_{I} \Omega_{Z}\right)}{\bar{\gamma}_{P} \Omega_{U} \Omega_{Y} \Omega_{Z}}\right)\right]+\Omega_{U}^{2} \Omega_{Y}^{2} e^{\frac{\bar{\gamma}_{I}\left(\Omega_{Z}-\Omega_{Y}\right)}{\bar{\gamma}_{P} \Omega_{V} \Omega_{Z}}} \\
& \times\left[\operatorname{Ei}\left(\frac{\bar{\gamma}_{I}\left(\Omega_{Y}-\Omega_{Z}\right)}{\bar{\gamma}_{P} \Omega_{V} \Omega_{Z}}\right)-\operatorname{Ei}\left(\frac{\left(a \Omega_{V}+\bar{\gamma}_{I} \Omega_{Y}\right)\left(\Omega_{Y}-\Omega_{Z}\right)}{\bar{\gamma}_{P} \Omega_{V} \Omega_{Y} \Omega_{Z}}\right)\right]\left[\Omega_{U}\left(b \Omega_{V}+\bar{\gamma}_{I} \Omega_{Y}\right)^{2}+\bar{\gamma}_{I} \Omega_{V} \Omega_{Z}\left(b \Omega_{V}+\bar{\gamma}_{I} \Omega_{Y}\right)\right. \\
& \left.\left.-\bar{\gamma}_{I} \bar{\gamma}_{P} \Omega_{V}^{2} \Omega_{Z}^{2}\right]\right\}+\frac{\bar{\gamma}_{P} \Omega_{U} \Omega_{V} \Omega_{Y}\left(b \Omega_{U} \Omega_{V}+\bar{\gamma}_{I} \Omega_{U} \Omega_{Y}+\bar{\gamma}_{I} \Omega_{V} \Omega_{Z}\right)}{\left(\Omega_{Y}-\Omega_{Z}\right)\left(a \Omega_{V}+\bar{\gamma}_{I} \Omega_{Y}\right)}\left\{e ^ { \frac { a ( \Omega _ { Y } - \Omega _ { Z } ) } { \overline { \gamma } _ { P } \Omega _ { Y } \Omega _ { Z } } } \left[a \Omega_{U} \Omega_{V} \Omega_{Z}\left(b \Omega_{V}+\bar{\gamma}_{I} \Omega_{Y}\right)\right.\right. \\
& \left.+\bar{\gamma}_{I} \Omega_{V} \Omega_{Z}^{2}\left(a \Omega_{V}+\bar{\gamma}_{I} \Omega_{Y}\right)+\bar{\gamma}_{I} \Omega_{U} \Omega_{Y}^{2}\left(b \Omega_{V}+\bar{\gamma}_{I} \Omega_{Y}\right)\right] \\
& \left.\left.-\left(a \Omega_{V}+\bar{\gamma}_{I} \Omega_{Y}\right)\left[\Omega_{U} \Omega_{Y}\left(b \Omega_{V}+\bar{\gamma}_{I} \Omega_{Y}\right)+\bar{\gamma}_{I} \Omega_{V} \Omega_{Z}^{2}\right]\right\}\right\} \\
& +\frac{\Omega_{Z} e^{-\frac{b}{\gamma P \Omega_{Z}}}}{\Omega_{Z}-\Omega_{Y}}\left(e^{\frac{a\left(\Omega_{Y}-\Omega_{Z}\right)}{\bar{\gamma} P \Omega_{Y} \Omega_{Z}}}-1\right)+\frac{\Omega_{Z} e^{-\frac{\left(\Omega_{Y}+\Omega_{Z}\right)\left(b \Omega_{U}+\bar{\gamma}_{I} \Omega_{Z}\right)}{\tilde{\gamma}_{P} \Omega_{U} \Omega_{Y} \Omega_{Z}}}}{\bar{\gamma}_{P} \Omega_{U} \Omega_{Y}\left(\Omega_{Y}-\Omega_{Z}\right)} \\
& \times\left\{\bar{\gamma}_{I}\left(\Omega_{Y}-\Omega_{Z}\right) e^{\frac{b}{\bar{\gamma}_{P} \Omega_{Z}}+\frac{\bar{\gamma}_{I}}{\bar{\gamma}_{P} \Omega_{U}}} \times\left[\operatorname{Ei}\left(\frac{\left(\Omega_{Y}-\Omega_{Z}\right)\left(a \Omega_{U}-b \Omega_{U}-\bar{\gamma}_{I} \Omega_{Z}\right)}{\bar{\gamma}_{P} \Omega_{U} \Omega_{Y} \Omega_{Z}}\right)\right.\right. \\
& \left.-\operatorname{Ei}\left(-\frac{\left(\Omega_{Y}-\Omega_{Z}\right)\left(b \Omega_{U}+\bar{\gamma}_{I} \Omega_{Z}\right)}{\bar{\gamma}_{P} \Omega_{U} \Omega_{Y} \Omega_{Z}}\right)\right]-\bar{\gamma}_{P} \Omega_{U} \Omega_{Y}\left(e^{\frac{a}{\bar{\gamma}_{P} \Omega_{Y}}}-e^{\frac{a}{\bar{\gamma}_{P} \Omega_{Z}}}\right) \\
& \left.\times e^{\frac{-a \Omega_{U}+b \Omega_{U}+\bar{\nu}_{I} \Omega_{Z}}{\bar{\gamma}_{P} \Omega_{U} \Omega_{Y}}}\right\}+\frac{e^{-\frac{b \Omega_{V}+\bar{\gamma}_{I} \Omega_{Z}}{\bar{\gamma}_{P} \Omega_{V} \Omega_{Z}}}}{\bar{\gamma}_{P} \Omega_{V} \Omega_{Z}\left(a \Omega_{V}+\bar{\gamma}_{I} \Omega_{Y}\right)} \\
& \times\left\{\frac{\bar{\gamma}_{P} \Omega_{V} \Omega_{Z}}{\Omega_{Y}-\Omega_{Z}}\left[e^{\frac{a\left(\Omega_{Y}-\Omega_{Z}\right)}{\bar{\gamma}_{P} \Omega_{Y} \Omega_{Z}}}\left(a \Omega_{V} \Omega_{Z}+\bar{\gamma}_{I} \Omega_{Y}^{2}\right)-\Omega_{Y}\left(a \Omega_{V}+\bar{\gamma}_{I} \Omega_{Y}\right)\right]\right. \\
& \left.-\bar{\gamma}_{I} \Omega_{Y}\left(a \Omega_{V}+\bar{\gamma}_{I} \Omega_{Y}\right) e^{\frac{\bar{\gamma}_{I}\left(\Omega_{Z}-\Omega_{Y}\right)}{\bar{\gamma}_{P} \Omega_{V} \Omega_{Z}}}\left[\operatorname{Ei}\left(\frac{\left(a \Omega_{V}+\bar{\gamma}_{I} \Omega_{Y}\right)\left(\Omega_{Y}-\Omega_{Z}\right)}{\bar{\gamma}_{P} \Omega_{V} \Omega_{Y} \Omega_{Z}}\right)-\operatorname{Ei}\left(\frac{\bar{\gamma}_{I}\left(\Omega_{Y}-\Omega_{Z}\right)}{\bar{\gamma}_{P} \Omega_{V} \Omega_{Z}}\right)\right]\right\}
\end{aligned}
$$

$\Theta_{5}\left(\tau_{1}\right) \triangleq \int_{0}^{\infty} F_{X}\left(\tau_{1}(w+2)\right) f_{W}(w) d w$

$$
\begin{aligned}
& =1-\frac{\Omega_{X} e^{-\frac{2 \tau_{1}}{\gamma_{P} \Omega_{X}}}}{\tau_{1} \Omega_{W}+\Omega_{X}}-\frac{e^{-\frac{\bar{\gamma}_{I}\left(\frac{1}{\Omega_{U}}+\frac{1}{\Omega_{V}}\right)+\frac{2 \tau_{1}}{\Omega_{X}}}{\bar{\gamma}_{P}}}}{\bar{\gamma}_{P} \tau_{1} \Omega_{U} \Omega_{V} \Omega_{W} \Omega_{X}\left(\tau_{1} \Omega_{W}+\Omega_{X}\right)\left(\tau_{1} \Omega_{U}\left(2 \Omega_{V}-\bar{\gamma}_{I} \Omega_{W}\right)+\bar{\gamma}_{I} \Omega_{V} \Omega_{X}\right)^{2}} \\
& \times\left\{\bar{\gamma}_{P} \tau_{1} \Omega_{U} \Omega_{V} \Omega_{W} \Omega_{X}\left(\tau_{1} \Omega_{U}\left(2 \Omega_{V}-\bar{\gamma}_{I} \Omega_{W}\right)+\bar{\gamma}_{I} \Omega_{V} \Omega_{X}\right)\right. \\
& \times\left(\tau_{1}^{2} \boldsymbol{\Omega}_{U} \boldsymbol{\Omega}_{W}\left(\bar{\gamma}_{I} \boldsymbol{\Omega}_{W}-2 \boldsymbol{\Omega}_{V}\right)+\bar{\gamma}_{I} \boldsymbol{\Omega}_{V} \boldsymbol{\Omega}_{X}^{2}\right)+\bar{\gamma}_{I}\left(\tau_{1} \boldsymbol{\Omega}_{W}+\boldsymbol{\Omega}_{X}\right) \\
& \times\left\{\Omega_{V}^{2} \Omega_{X}^{2} e^{\frac{\left(\tau_{1} \Omega_{W}+\Omega_{X}\right)\left(\bar{y}_{I} \Omega_{X}+2 \tau_{1} \Omega_{U}\right)}{\bar{\gamma}_{P} \tau_{1} \Omega_{U} \Omega_{W} \Omega_{X}}}\left[\Omega_{V}\left(\bar{\gamma}_{I} \Omega_{X}+2 \tau_{1} \Omega_{U}\right)^{2}-\bar{\gamma}_{I} \tau_{1} \Omega_{U} \Omega_{W}\left(\bar{\gamma}_{I} \Omega_{X}+\tau_{1} \Omega_{U}\left(\bar{\gamma}_{P} \Omega_{W}+2\right)\right)\right]\right. \\
& \times \operatorname{Ei}\left(-\frac{\left(\tau_{1} \Omega_{W}+\Omega_{X}\right)\left(2 \tau_{1} \Omega_{U}+\bar{\gamma}_{I} \Omega_{X}\right)}{\bar{\gamma}_{P} \tau_{1} \Omega_{U} \Omega_{W} \Omega_{X}}\right)-\tau_{1}^{2} \Omega_{U}^{2} \Omega_{W}^{2} e^{\frac{\bar{\gamma}_{I}\left(\tau_{1} \Omega_{W}+\Omega_{X}\right)}{\gamma_{P} \Omega_{V} \Omega_{X}}}\left[-\bar{\gamma}_{I} \bar{\gamma}_{P} \Omega_{V}^{2} \Omega_{X}^{2}+\tau_{1}^{2} \Omega_{U}\left(\bar{\gamma}_{I} \Omega_{W}-2 \Omega_{V}\right)^{2}\right. \\
& \left.\left.\left.+\bar{\gamma}_{I} \tau_{1} \Omega_{V} \Omega_{X}\left(2 \Omega_{V}-\bar{\gamma}_{I} \Omega_{W}\right)\right] \operatorname{Ei}\left(-\frac{\bar{\gamma}_{I}\left(\tau_{1} \Omega_{W}+\Omega_{X}\right)}{\bar{\gamma}_{P} \Omega_{V} \Omega_{X}}\right)\right\}\right\}+\frac{\Omega_{X} e^{-\frac{\bar{\gamma}_{I} \Omega_{X}+2 \tau_{1} \Omega_{U}}{\bar{\gamma}_{P} \Omega_{U} \Omega_{X}}}}{\bar{\gamma}_{P} \tau_{1} \Omega_{U} \Omega_{W}\left(\tau_{1} \Omega_{W}+\Omega_{X}\right)} \\
& \times\left[\bar{\gamma}_{P} \tau_{1} \Omega_{U} \Omega_{W}+\bar{\gamma}_{I}\left(\tau_{1} \Omega_{W}+\Omega_{X}\right) e^{\frac{\left(\tau_{1} \Omega_{W}+\Omega_{X}\right)\left(\bar{\gamma}_{I} \Omega_{X}+2 \tau_{1} \Omega_{U}\right)}{\bar{\gamma}_{P} \tau_{1} \Omega_{U} \Omega_{W} \Omega_{X}}} \operatorname{Ei}\left(-\frac{\left(\tau_{1} \Omega_{W}+\Omega_{X}\right)\left(2 \tau_{1} \Omega_{U}+\bar{\gamma}_{I} \Omega_{X}\right)}{\bar{\gamma}_{P} \tau_{1} \Omega_{U} \Omega_{W} \Omega_{X}}\right)\right] \\
& -\frac{\tau_{1} \Omega_{W} e^{-\frac{\bar{y}_{I} \Omega_{X}+2 \tau_{1} \Omega_{V}}{\bar{\gamma}_{P} \Omega_{V} \Omega_{X}}}}{\bar{\gamma}_{P} \Omega_{V} \Omega_{X}\left(\tau_{1} \Omega_{W}+\Omega_{X}\right)} \times\left[\bar{\gamma}_{P} \Omega_{V} \Omega_{X}+\bar{\gamma}_{I}\left(\tau_{1} \Omega_{W}+\Omega_{X}\right) e^{\frac{\bar{\gamma}_{I}\left(\tau_{1} \Omega_{W}+\Omega_{X}\right)}{\gamma_{P} \Omega_{V} \Omega_{X}}} \operatorname{Ei}\left(-\frac{\bar{\gamma}_{I}\left(\tau_{1} \Omega_{W}+\Omega_{X}\right)}{\bar{\gamma}_{P} \Omega_{V} \Omega_{X}}\right)\right]
\end{aligned}
$$

with $\Theta_{3}\left(\tau_{1}\right)$ and $\Theta_{4}(a, b)$ being given by (45), shown at the bottom of the previous page, and (46), shown at the top of this page, respectively. Finally, by combining (37)-(41) into (13) and after some algebraic manipulations, we arrive at a new useful closed-form approximation to the outage probability of an underlay CRN operating under the proposed adaptive transmission scheme, given as in (17). 


\section{APPENDIX E}

\section{PROOF OF THE THEOREM 3}

For a scenario in which the maximum transmit system power at the secondary network, $P_{\mathrm{T}}$, is varied independently of the interference power constraint at the primary receiver, $I$, we have that all the terms proportional to $1 / \bar{\gamma}_{P}$ go to zero as $P_{\mathrm{T}}$ becomes larger in the high-SNR regime (vide definition for $\bar{\gamma}_{P}$ in Lemma 1). In addition, from the Maclaurin series expansion of the exponential function [47, eq. (0.318.2)], it follows that $e^{-\lambda} \simeq 1-\lambda$ when $\lambda \rightarrow 0$. Using this into (17) and neglecting the high-order terms with respect to $1 / \bar{\gamma}_{P}$, after some lengthy manipulations, an asymptotic closed-form expression for the outage probability of the proposed scheme can be obtained as in (18).

\section{REFERENCES}

[1] C.-X. Wang et al., "Cellular architecture and key technologies for 5G wireless communication networks," IEEE Commun. Mag., vol. 52, no. 2, pp. 122-130, Feb. 2014.

[2] J. G. Andrews et al., "What will 5G be?" IEEE J. Sel. Areas Commun., vol. 32, no. 6, pp. 1065-1082, Jun. 2014.

[3] J. Mitola, III, and G. Q. Maguire, Jr., "Cognitive radio: Making software radios more personal," IEEE Pers. Commun., vol. 6, no. 4, pp. 13-18, Apr. 1999

[4] S. Haykin, "Cognitive radio: Brain-empowered wireless communications," IEEE J. Sel. Areas Commun., vol. 23, no. 2, pp. 201-220, Feb. 2005

[5] B. Wang and K. J. R. Liu, "Advances in cognitive radio networks: A survey," IEEE J. Sel. Topics Signal Process., vol. 5, no. 1, pp. 5-23, Feb. 2011.

[6] W. Krenik, A. M. Wyglinski, and L. E. Doyle, "Guest editorialCognitive radios for dynamic spectrum access," IEEE Commun. Mag., vol. 45, no. 5, pp. 64-65, May 2007.

[7] A. Goldsmith, S. A. Jafar, I. Maric, and S. Srinivasa, "Breaking spectrum gridlock with cognitive radios: An information theoretic perspective," Proc. IEEE, vol. 97, no. 5, pp. 894-914, Apr. 2009.

[8] A. Sendonaris, E. Erkip, and B. Aazhang, "User cooperation diversityPart I: System description," IEEE Trans. Commun., vol. 51, no. 11, pp. 1927-1938, Nov. 2003.

[9] J. N. Laneman, D. N. C. Tse, and G. W. Wornell, "Cooperative diversity in wireless networks: Efficient protocols and outage behavior," IEEE Trans. Inf. Theory, vol. 50, no. 12, pp. 3062-3080, Dec. 2004.

[10] M. Dohler and Y. Li, Cooperative Communications: Hardware, Channel and PHY. West Sussex, U.K.: Wiley, 2010.

[11] D. Tse and P. Viswanath, Fundamentals of Wireless Communication. Cambridge, UK: Cambridge Univ. Press, 2005.

[12] M. Duarte and A. Sabharwal, "Full-duplex wireless communications using off-the-shelf radios: Feasibility and first results," in Proc. 44th Asilomar Conf. Signals, Syst. Comput., Pacific Grove, CA, USA, Nov. 2010, pp. 1558-1562.

[13] T. Riihonen, S. Werner, and R. Wichman, "Residual self-interference in full-duplex MIMO relays after null-space projection and cancellation," in Proc. 44th Asilomar Conf. Signals, Syst. Comput., Pacific Grove, CA, USA, Nov. 2010, pp. 653-657.

[14] T. Riihonen, S. Werner, and R. Wichman, "Mitigation of loopback selfinterference in full-duplex MIMO relays," IEEE Trans. Signal Process. vol. 59, no. 12, pp. 5983-5993, Dec. 2011.

[15] E. Everett, A. Sahai, and A. Sabharwal, "Passive self-interference suppression for full-duplex infrastructure nodes," IEEE Trans. Wireless Commun., vol. 13, no. 2, pp. 680-694, Jan. 2014.

[16] M. Duarte et al., "Design and characterization of a full-duplex multiantenna system for WiFi networks," IEEE Trans. Veh. Technol., vol. 63, no. 3, pp. $1160-1177$, Mar. 2014

[17] T. Riihonen and R. Wichman, "Analog and digital self-interference cancellation in full-duplex MIMO-OFDM transceivers with limited resolution in A/D conversion," in Proc. 46th Asilomar Conf. Signals, Syst. Comput., Pacific Grove, CA, USA, Nov. 2012, pp. 45-49.

[18] M. Duarte, C. Dick, and A. Sabharwal, "Experiment-driven characterization of full-duplex wireless systems," IEEE Trans. Wireless Commun., vol. 11 , no. 12 , pp. 4296-4307, Dec. 2012.
[19] Y. Hua, Y. Ma, P. Liang, and A. Cirik, "Breaking the barrier of transmission noise in full-duplex radio," in Proc. IEEE MILCOM, San Diego, CA, USA, Nov. 2013, pp. 1558-1563.

[20] E. Ahmed and A. M. Eltawil, "All-digital self-interference cancellation technique for full-duplex systems," IEEE Trans. Wireless Commun., vol. 14, no. 7, pp. 3519-3532, Jul. 2015.

[21] T. Riihonen, S. Werner, and R. Wichman, "Hybrid full-duplex/halfduplex relaying with transmit power adaptation," IEEE Trans. Wireless Commun., vol. 10, no. 9, pp. 3074-3085, Sep. 2011.

[22] I. Krikidis, H. A. Suraweera, P. J. Smith, and C. Yuen, "Full-duplex relay selection for amplify-and-forward cooperative networks," IEEE Trans. Wireless Commun., vol. 11, no. 12, pp. 4381-4393, Dec. 2012.

[23] H. Alves, D. B. da Costa, R. D. Souza, and M. Latva-aho, "Performance of block-Markov full duplex relaying with self interference in Nakagami-m fading," IEEE Wireless Commun. Lett., vol. 2, no. 3 , pp. 311-314, Jun. 2013.

[24] M. G. Khafagy, A. Ismail, M.-S. Alouini, and S. Aïssa, "Efficient cooperative protocols for full-duplex relaying over Nakagami- $m$ fading channels," IEEE Trans. Wireless Commun., vol. 14, no. 6, pp. 3456-3470, Jun. 2015.

[25] C. Zhong, T. Ratnarajah, and K.-K. Wong, "Outage analysis of decodeand-forward cognitive dual-hop systems with the interference constraint in Nakagami- $m$ fading channels," IEEE Trans. Veh. Technol., vol. 60, no. 6, pp. 2875-2879, Jul. 2011.

[26] T. Q. Duong, V. N. Q. Bao, and H.-J. Zepernick, "Exact outage probability of cognitive AF relaying with underlay spectrum sharing," Electron. Lett., vol. 47, no. 17, pp. 1001-1002, Aug. 2011.

[27] J. Lee, H. Wang, J. G. Andrews, and D. Hong, "Outage probability of cognitive relay networks with interference constraints," IEEE Trans. Wireless Commun., vol. 10, no. 2, pp. 390-395, Feb. 2011.

[28] L. Luo, P. Zhang, G. Zhang, and J. Qin, "Outage performance for cognitive relay networks with underlay spectrum sharing," IEEE Commun. Lett., vol. 15, no. 7, pp. 710-712, Jul. 2011.

[29] X. Zhang, Y. Zhang, Z. Yan, J. Xing, and W. Wang, "Performance analysis of cognitive relay networks over Nakagami- $m$ fading channels," IEEE J. Sel. Areas Commun., vol. 33, no. 5, pp. 865-877, May 2015.

[30] D. B. da Costa, M. Elkashlan, P. L. Yeoh, N. Yang, and M. D. Yacoub, "Dual-hop cooperative spectrum sharing systems with multi-primary users and multi-secondary destinations over Nakagami- $m$ fading," in Proc. IEEE 23rd Int. Symp. PIMRC, Sydney, NSW, Australia, Sep. 2012, pp. $1577-1581$.

[31] E. E. Benítez Olivo, D. P. Moya Osorio, D. B. da Costa, and J. C. S. Santos Filho, "Outage performance of spectrally efficient schemes for multiuser cognitive relaying networks with underlay spectrum sharing," IEEE Trans. Wireless Commun., vol. 13, no. 12, pp. 6629-6642, Dec. 2014.

[32] P. L. Yeoh, M. Elkashlan, T. Q. Duong, N. Yang, and D. B. da Costa, "Transmit antenna selection for interference management in cognitive relay networks," IEEE Trans. Veh. Technol., vol. 63, no. 7, pp. 3250-3262, Sep. 2014.

[33] Y. Deng, M. Elkashlan, P. L. Yeoh, N. Yang, and R. K. Mallik, "Cognitive MIMO relay networks with generalized selection combining," IEEE Trans. Wireless Commun., vol. 13, no. 9, pp. 4911-4922, Sep. 2014.

[34] H. Kim, S. Lim, H. Wang, and D. Hong, "Optimal power allocation and outage analysis for cognitive full duplex relay systems," IEEE Trans. Wireless Commun., vol. 11, no. 10, pp. 3754-3765, Oct. 2012.

[35] S. B. Mafra, H. Alves, D. B. da Costa, R. D. Souza, E. M. G. Fernandez, and M. Latva-aho, "On the performance of cognitive full-duplex relaying under spectrum sharing constraints," EURASIP J. Wireless Commun. Netw., vol. 2015, no. 1, pp. 1-13, Jun. 2015.

[36] Y. Deng, K. J. Kim, T. Q. Duong, M. Elkashlan, G. K. Karagiannidis, and A. Nallanathan, "Full-duplex spectrum sharing in cooperative single carrier systems," in Proc. IEEE WCNC, New Orleans, LA, USA, Mar. 2015, pp. 25-30.

[37] A. Carleial, "Multiple-access channels with different generalized feedback signals," IEEE Trans. Inf. Theory, vol. 28, no. 6, pp. 841-850, Nov. 1982.

[38] F. M. J. Willems, "Information theoretical results for the discrete memoryless multiple access channel," Ph.D. dissertation, Dept. Math. Katholieke Univ. Leuven, Leuven, Belgium, Oct. 1982.

[39] G. Kramer, M. Gastpar, and P. Gupta, "Cooperative strategies and capacity theorems for relay networks," IEEE Trans. Inf. Theory, vol. 51, no. 9, pp. 3037-3063, Sep. 2005.

[40] T. Kwon, S. Lim, S. Choi, and D. Hong, "Optimal duplex mode for DF relay in terms of the outage probability," IEEE Trans. Veh. Technol., vol. 59, no. 7, pp. 3628-3634, Sep. 2010. 
[41] Q. Zhao, S. Geirhofer, L. Tong, and B. M. Sadler, "Opportunistic spectrum access via periodic channel sensing," IEEE Trans. Signal Process., vol. 56, no. 2, pp. 785-796, Feb. 2008.

[42] H. Ding, J. Ge, D. B. da Costa, and Z. Jiang, "Link selection schemes for selection relaying systems with transmit beamforming: New and efficient proposals from a distributed concept," IEEE Trans. Veh. Technol., vol. 61, no. 2, pp. 533-552, Feb. 2012.

[43] G. S. Smith, "A direct derivation of a single-antenna reciprocity relation for the time domain," IEEE Trans. Antennas Propag., vol. 52, no. 6, pp. 1568-1577, Jun. 2004.

[44] A. Ghasemi and E. S. Sousa, "Fundamental limits of spectrum-sharing in fading environments," IEEE Trans. Wireless Commun., vol. 6, no. 2, pp. 649-658, Feb. 2007.

[45] J. M. Peha, "Approaches to spectrum sharing," IEEE Commun. Mag., vol. 43, no. 2, pp. 10-12, Feb. 2005.

[46] A. Papoulis and S. U. Pillai, Probability, Random Variables and Stochastic Processes, 4th ed. New York, NY, USA: McGraw-Hill, 2002.

[47] I. S. Gradshteyn and I. M. Ryzhik, Table of Integrals, Series, and Products, 7th ed. New York, NY, USA: Academic, 2007.

[48] L. Zheng and D. N. C. Tse, "Diversity and multiplexing: A fundamental tradeoff in multiple-antenna channels," IEEE Trans. Inf. Theory, vol. 49, no. 5, pp. 1073-1096, May 2003.

[49] E. W. Weisstein. (2015). Sifting Property. [Online]. Available: http:// mathworld.wolfram.com/SiftingProperty.html

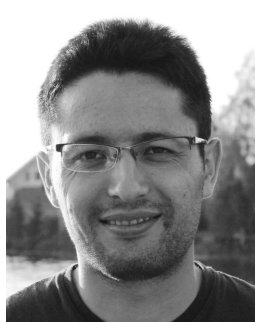

Edgar Eduardo Benítez Olivo received the B.Sc. degree in electrical and telecommunications engineering from Armed Forces University-ESPE, Sangolquí, Ecuador, in 2008, and the M.Sc. and $\mathrm{Ph} . \mathrm{D}$. degrees in electrical engineering from the University of Campinas, Campinas, Brazil, in 2011 and 2015, respectively. In 2014, he was a Visiting Researcher with the Centre for Wireless Communications, University of Oulu, Finland. He is currently an Assistant Professor with the School of Telecommunications Engineering, São Paulo State University, São Jõao da Boa Vista, Brazil. His research interests lie in the area of wireless communications, with a current focus on emerging technologies toward $5 \mathrm{G}$ wireless networks.

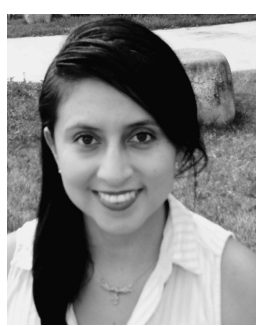

Diana Pamela Moya Osorio was born in Quito, Ecuador. She received the B.Sc. degree in electrical and telecommunications engineering from Armed Forces University-ESPE, Sangolquí, Ecuador, in 2008, and the M.Sc. and D.Sc. degrees in electrical engineering from the University of Campinas, Campinas, Brazil, in 2011 and 2015, respectively. She is currently an Assistant Professor with the Department of Electrical Engineering, Federal University of São Carlos, São Carlos, Brazil. Her research interests include wireless communications in general, cooperative communications, cognitive radio systems, physical layer security, and $5 \mathrm{G}$ technologies.

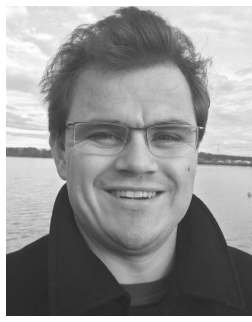

Hirley Alves received the B.Sc., M.Sc., and D.Sc. degrees in electrical engineering from the Federal University of Technology-Paraná, Brazil, in 2010, 2011, and 2015, respectively, and the D.Sc. degree from the University of Oulu, in 2015. He is currently a Researcher with the Centre for Wireless Communications, Oulu. His current research focuses on ultrareliable communication on machine type networks, performance analysis of full-duplex relaying networks, physical layer security, 5G networks, and smart grids.

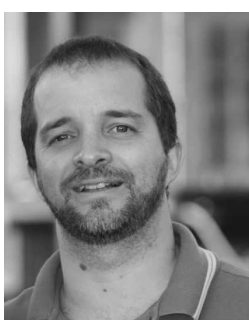

José Cândido Silveira Santos Filho (M'09) received the B.Sc., M.Sc., and Ph.D. degrees from the School of Electrical and Computer Engineering (FEEC), University of Campinas (UNICAMP), Campinas, Brazil, in 2001, 2003, and 2006, respectively, all in electrical engineering. He was ranked first in his undergraduate program. From 2006 to 2009, he was a Post-Doctoral Fellow with the Wireless Technology Laboratory, FEEC-UNICAMP. He is currently an Assistant Professor with FEEC-UNICAMP. Since 2011, he has regularly consulted for Bradar Indústria S.A., a branch of Embraer Defense and Security, in the development of innovative radar techniques. He has authored over 70 technical papers, about half of which in international journals, and has served as a Reviewer for many journals and conferences. His research areas include wireless communications and radar systems. His Ph.D. Thesis received an Honorary Mention by the Brazilian Ministry of Education (CAPES) in the 2007 CAPES Thesis Contest.

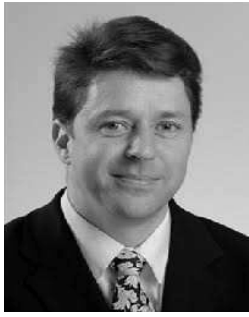

Matti Latva-aho was born in Kuivaniemi, Finland, in 1968. He received the M.Sc., Lic.Tech., and Dr.Tech. (Hons.) degrees from the University of Oulu, Finland, in 1992, 1996, and 1998, respectively, all in electrical engineering. From 1992 to 1993, he was a Research Engineer with Nokia Mobile Phones, Oulu, Finland. From 1994 to 1998, he was a Research Scientist with the Telecommunication Laboratory and the Centre for Wireless Communications, University of Oulu. He was the Director of the Centre for Wireless Communications with the University of Oulu from 1998 to 2006. He is currently the Chair of the Department of Communications Engineering and a Professor of Digital Transmission Techniques with the University of Oulu. 